TRANSACTIONS OF THE

AMERICAN MATHEMATICAL SOCIETY

Volume 357, Number 3, Pages 875-900

S 0002-9947(04)03708-0

Article electronically published on October 19, 2004

\title{
ON THE DEGENERATE BELTRAMI EQUATION
}

\author{
V. GUTLYANSKII, O. MARTIO, T. SUGAWA, AND M. VUORINEN
}

\begin{abstract}
We study the well-known Beltrami equation under the assumption that its measurable complex-valued coefficient $\mu(z)$ has the norm $\|\mu\|_{\infty}=$ 1. Sufficient conditions for the existence of a homeomorphic solution to the Beltrami equation on the Riemann sphere are given in terms of the directional dilatation coefficients of $\mu$. A uniqueness theorem is also proved when the singular set $\operatorname{Sing}(\mu)$ of $\mu$ is contained in a totally disconnected compact set with an additional thinness condition on $\operatorname{Sing}(\mu)$.
\end{abstract}

\section{INTRODUCTION}

The analytic theory of plane quasiconformal mappings $f$ is based on the Beltrami partial differential equation

$$
f_{\bar{z}}=\mu(z) f_{z} \quad \text { a.e. }
$$

with the complex-valued measurable coefficient $\mu$ satisfying the uniform ellipticity assumption $\|\mu\|_{\infty}<1$. In the case $|\mu(z)|<1$ a.e. in $\mathbb{C}$ and $\|\mu\|_{\infty}=1$, (1.1) is called a degenerate Beltrami equation and the structure of the solutions heavily depends on the degeneration of $\mu$. In this article, unless otherwise stated, by a Beltrami coefficient in a domain $\Omega$ we mean a complex-valued measurable function $\mu$ in $\Omega$ such that $|\mu|<1$ a.e. in $\Omega$, and by a solution to the Beltrami equation (1.1) in a domain $\Omega$ we mean a function $f$ in the Sobolev space $W_{\text {loc }}^{1,1}(\Omega)$ whose partial derivatives satisfy (1.1) in $\Omega$. Then $f$ is called $\mu$-conformal in $\Omega$. The measurable Riemann mapping theorem (cf. [1]) states that given a measurable function $\mu$ in the plane $\mathbb{C}$ with $\|\mu\|_{\infty}<1$ there is a quasiconformal homeomorphism $f: \mathbb{C} \rightarrow \mathbb{C}$, $f \in W_{\text {loc }}^{1,2}(\mathbb{C})$, satisfying (1.1). Contrary to this, the degenerate Beltrami equation need not have a homeomorphic solution, and a solution, if it exists, need not be unique. See, for instance, 12. Therefore, in order to obtain existence or uniqueness results, some extra constraints must be imposed on $\mu$.

The degeneration of $\mu$ is usually expressed in terms of the pointwise maximal dilatation function

$$
K(z)=K_{\mu}(z)=\frac{1+|\mu(z)|}{1-|\mu(z)|} .
$$

This takes into account the absolute value of $\mu$ only. In this paper we show that for sharper results the argument of $\mu(z)$ should also be considered. For example, consider the Beltrami coefficients $\mu_{1}(z)=(1-|z|) z / \bar{z}$ and $\mu_{2}(z)=(|z|-1) z / \bar{z}$

Received by the editors February 11, 2002.

2000 Mathematics Subject Classification. Primary 30C62.

The third author was partially supported by the Academy of Finland and the JSPS while carrying out the present paper. 
defined in the unit disk $\mathbb{D}$. It is immediate that $\left\|\mu_{j}\right\|_{\infty}=1, j=1,2$, and $K_{\mu_{1}}(z)=$ $K_{\mu_{2}}(z)$ whenever $z \in \mathbb{D} \backslash\{0\}$. The radial stretching $f_{1}: \mathbb{D} \rightarrow \mathbb{D}$, defined by

$$
f_{1}(z)=\frac{z}{|z|^{2}} e^{2(1-1 /|z|)}
$$

for $z \in \mathbb{D} \backslash\{0\}, f_{1}(0)=0$, is $\mu_{1}$-conformal. The second radial stretching

$$
f_{2}(z)=\frac{z}{|z|(2-|z|)}
$$

in the punctured disk $\mathbb{D} \backslash\{0\}$ is $\mu_{2}$-conformal and has the cavitation effect since it maps $\mathbb{D} \backslash\{0\}$ homeomorphically onto the annulus $1 / 2<|z|<1$. Actually, the continuous solution to the Beltrami equation with $\mu=\mu_{2}$ is unique up to the post-composition of analytic functions (cf. Proposition 4.1 below), and hence the cavitation is inevitable in this case. Thus the cavitation problem requires more precise information on $\mu$ than merely on $|\mu|$.

To study the aforementioned problem we employ the angular dilatation coefficient $D_{\mu, z_{0}}$ (see (2.13) below) to take into account an effect of the argument of $\mu$ as well. On one hand, it allows us to prove the existence of a homeomorphic solution $f$ to the Beltrami equation (1.1) for a given Beltrami coefficient $\mu$ with $\|\mu\|_{\infty}=1$ provided that $D_{\mu, z_{0}}$ satisfies a local integrability condition for each $z_{0}$; see Theorem 3.5. We also obtain an estimate for the modulus of continuity of $f$. On the other hand, we establish a uniqueness theorem for the solution of (1.1) in the case when the singular sets of $\mu$ are totally disconnected compacta with certain geometric condition involving $D_{\mu, z_{0}}$; see Theorem 4.3. The modulus estimate for annuli in Lemma 2.19 in terms of the integral means of the angular dilatation coefficients plays a crucial role in the proof of the existence and uniqueness results. A normal family argument is also used; see Propositions 2.1] and 2.3.

The idea of employing $\mu$ instead of $|\mu|$ in the study of some regularity problems for quasiconformal mappings is due to Andreian Cazacu 2$]$ and Reich and Walczak 20]. O. Lehto [14, [15] was the first who considered the degenerate Beltrami equation from this point of view.

The degeneration of $\mu$ in terms of $|\mu(z)|$ or $K_{\mu}(z)$ has recently been extensively studied. This is due to the close connection of $f$ in (1.1) to the solutions of elliptic partial differential equations. For the earlier studies of $\mu$-homeomorphisms we refer to [4, 5], [19] and [18]. The results of Pesin [19] have been substantially extended by Brakalova and Jenkins [6]. For the recent deep theorems on the existence and uniqueness of $\mu$-homeomorphisms see Iwaniec and Martin [12], who extended the well-known results of David [8] and Tukia [24], and see also [22], 21], 7] and the references therein.

Let us indicate a couple of features of our main results. First, by virtue of adoption of the angular dilatation coefficients, the existence theorem and its primitive, Theorem 2.15] cover many cases when $K_{\mu}$ fails to satisfy known integrability conditions; see, for instance, Examples 3.28 and 3.29. Even the case when the singular set of $\mu$ consists of finitely many points is of independent interest; see Section 3 .

1.5. Theorem. Let $\mu(z)$ be a Beltrami coefficient in $\widehat{\mathbb{C}}$ such that the set of singularity $\operatorname{Sing}(\mu)$ (see Remark 2.18) consists of finitely many points. Then there exists a homeomorphism $f: \widehat{\mathbb{C}} \rightarrow \widehat{\mathbb{C}}$ which is locally quasiconformal in $\widehat{\mathbb{C}} \backslash \operatorname{Sing}(\mu)$ and whose complex dilatation $\mu_{f}$ satisfies $\left|\mu_{f}(z)\right|=|\mu(z)|$ a.e. 
Secondly, Theorem 3.5 applied to the classical setting involving $K_{\mu}$ only gives the following result.

1.6. Theorem. Suppose that a Beltrami coefficient $\mu$ on $\mathbb{C}$ satisfies

$$
\iint_{\mathbb{C}} e^{H\left(K_{\mu}(z)\right)} \frac{d x d y}{\left(1+|z|^{2}\right)^{2}}<+\infty
$$

for a measurable function $H:[1,+\infty) \rightarrow \mathbb{R}$ for which there exist an integer $n \geq 1$ and numbers $c>0, \alpha \in(-\infty, 1]$ such that

$$
H(t) \geq c t /(\log t)\left(\log _{2} t\right) \cdots\left(\log _{n-1} t\right)\left(\log _{n} t\right)^{\alpha}
$$

for large $t$. Then there exists a homeomorphic solution $f: \mathbb{C} \rightarrow \mathbb{C}$ to the Beltrami equation with $\mu$ such that $f \in W_{\text {loc }}^{1, q}(\mathbb{C})$ for every $1 \leq q<2$ and $f^{-1} \in W_{\mathrm{loc}}^{1,2}(\mathbb{C})$. Moreover, for $\alpha<1, n \geq 2$ and for $\alpha \in[0,1)$ if $n=1, f$ satisfies the inequality

$$
\left|f(z)-f\left(z_{0}\right)\right| \leq C \exp \left(\frac{-c}{2(1-\alpha)}\left(\log _{n+1} \frac{1}{\left|z-z_{0}\right|}\right)^{1-\alpha}\right), \quad\left|z-z_{0}\right|<\delta_{0},
$$

where the constants $C>0$ and $\delta_{0}>0$ are locally uniformly bounded above and below, respectively. If $n=1$ and $\alpha<0$, the above inequality still holds with the constant $c /(2(1-\alpha))$ being replaced by any larger number.

Here, $\log _{n}$ denotes the iterated logarithm; see Section 5. Examples show that this result is close of being optimal. Indeed, for each $n$, one cannot take $\alpha>1$ in the integrability condition above. Moreover, the constant $c /(2(1-\alpha))$ and the exponent $1-\alpha$ in the above estimate for modulus of continuity cannot be replaced by any smaller constants.

This paper is organized as follows. In Section 2, we give modulus estimates for ring domains and establish normality theorems for some families of homeomorphisms in order to prove Theorem 2.15. The existence theorems, modulus of continuity estimates and examples are collected in Section 3. Section 4 is devoted to uniqueness theorems. Theorem 1.6 is proved in Section 5. This section also contains a treatment of iterated logarithms.

\section{Sequences of SELF-HOMEOMORPhisms}

The degenerate Beltrami equation need not have a homeomorphic solution nor even a nonconstant solution. A usual approach for the existence of a solution is to consider a sequence $f_{n}$ of quasiconformal homeomorphisms satisfying (1.1) with the Beltrami coefficients $\mu_{n},\left\|\mu_{n}\right\|_{\infty}<1$, such that $\mu_{n} \rightarrow \mu$ a.e. and then to use a normal family argument to obtain a limit mapping $f$. Some conditions must be imposed on $\mu$ in order to pick a converging subsequence.

In the following we introduce a modulus method to study normal families of homeomorphisms. This will be employed to solve the degenerated Beltrami equation; however, the method is of independent interest.

We introduce some notation. We denote the Euclidean distance and the spherical distance between $z$ and $w$ by

$$
d(z, w)=|z-w| \quad \text { and } \quad d^{\sharp}(z, w)=|z-w| / \sqrt{\left(1+|z|^{2}\right)\left(1+|w|^{2}\right)},
$$

respectively. Also we denote by $A\left(z_{0}, r, R\right)$ and by $A^{\sharp}\left(z_{0}, r, R\right)$ the (circular) annuli in the Euclidean and the spherical metric, respectively, i.e.,

$$
A\left(z_{0}, r, R\right)=B\left(z_{0}, R\right) \backslash \bar{B}\left(z_{0}, r\right) \quad \text { and } \quad A^{\sharp}\left(z_{0}, r, R\right)=B^{\sharp}\left(z_{0}, R\right) \backslash \bar{B}^{\sharp}\left(z_{0}, r\right)
$$


for $z_{0} \in \widehat{\mathbb{C}}$ and $0 \leq r<R$, where $B\left(z_{0}, r\right)=\left\{z \in \mathbb{C}:\left|z-z_{0}\right|<r\right\}$ and $B^{\sharp}\left(z_{0}, r\right)=$ $\left\{z \in \widehat{\mathbb{C}}: d^{\sharp}\left(z, z_{0}\right)<r\right\}$. Here, in the case when $z_{0}=\infty$, we set $B(\infty, r)=\{z \in$ $\widehat{\mathbb{C}}:|z|>1 / r\}$, and hence, $A(\infty, r, R)=A(0,1 / R, 1 / r)$. In the sequel, for subsets $E, E_{0}, E_{1}$ of $\widehat{\mathbb{C}}$, $\operatorname{diam} E$ and $\operatorname{dist}\left(E_{0}, E_{1}\right)$ stand for the diameter of $E$ and the distance between $E_{0}$ and $E_{1}$, respectively, measured in the Euclidean metric $d$. Similarly, $\operatorname{diam}^{\sharp} E$ and $\operatorname{dist}^{\sharp}\left(E_{0}, E_{1}\right)$ stand for those measured in the spherical metric $d^{\sharp}$. We also denote by $\mathcal{A}$ and $\mathcal{A}^{\sharp}$ the two-dimensional Lebesgue measure and the spherical measure, respectively, i.e., $\mathcal{A}(E)=\iint_{E} d x d y$ and $\mathcal{A}^{\sharp}(E)=\iint_{E}\left(1+|z|^{2}\right)^{-2} d x d y$.

A doubly connected domain is called a ring domain. The modulus $m$ of a ring domain $A$ is the number such that $A$ is conformally equivalent to $\left\{1<|z|<e^{m}\right\}$ and will be denoted by $\bmod A$. When $A$ is conformally equivalent to $\mathbb{C}^{*}=\mathbb{C} \backslash\{0\}$, we define $\bmod A=\infty$. A non-negative function $\rho(z, r, R)$ in $(z, r, R) \in \widehat{\mathbb{C}} \times(0,+\infty) \times$ $(0,+\infty), r<R$, will be called a modulus constraint if $\rho\left(z_{0}, r, R\right) \rightarrow+\infty$ as $r \rightarrow 0$ for any fixed $R \in(0,+\infty)$ and $z_{0} \in \widehat{\mathbb{C}}$.

We denote by $\mathcal{H}_{\rho}$ the family of all normalized homeomorphisms $f: \widehat{\mathbb{C}} \rightarrow \widehat{\mathbb{C}}$ such that the condition

$$
\bmod f\left(A\left(z_{0}, r, R\right)\right) \geq \rho\left(z_{0}, r, R\right)
$$

holds for all $z_{0} \in \widehat{\mathbb{C}}$ and $r, R \in(0,+\infty)$ with $r<R$. Here and hereafter, a homeomorphism $f: \widehat{\mathbb{C}} \rightarrow \widehat{\mathbb{C}}$ is said to be normalized if $f$ fixes 0,1 and $\infty$. Note that the above condition is not Möbius invariant.

Note also that, without changing the family $\mathcal{H}_{\rho}$, we may always assume the monotonicity condition $\rho\left(z_{0}, r_{1}, R_{1}\right) \leq \rho\left(z_{0}, r_{2}, R_{2}\right)$ for $z_{0} \in \widehat{\mathbb{C}}$ and $r_{2} \leq r_{1}<R_{1} \leq R_{2}$.

Similarly, a non-negative function $\rho^{\sharp}(z, r, R)$ in $(z, r, R) \in \widehat{\mathbb{C}} \times(0,1) \times(0,1), r<$ $R$, will be called a spherical modulus constraint if $\rho^{\sharp}(z, r, R) \rightarrow+\infty$ as $r \rightarrow 0$ for any fixed $R \in(0,1)$ and $z \in \widehat{\mathbb{C}}$. We let $\mathcal{H}_{\rho^{\sharp}}^{\sharp}$ be the family of all normalized selfhomeomorphisms $f$ of $\widehat{\mathbb{C}}$ such that $\bmod f\left(A^{\sharp}\left(z_{0}, r, R\right)\right) \geq \rho^{\sharp}\left(z_{0}, r, R\right)$ holds for all $0<r<R<1$ and $z_{0} \in \widehat{\mathbb{C}}$.

A family of continuous maps from a domain $\Omega$ into $\widehat{\mathbb{C}}$ is said to be normal or compact if it is relatively compact or compact, respectively, with respect to the topology of local uniform convergence.

The following propositions are similar to a result in [15] where stronger assumptions were used (see also Lemma 1 in Section 4 of [6]).

2.1. Proposition. Let $\rho^{\sharp}$ be a spherical modulus constraint. Then

1) $\mathcal{H}_{\rho^{\sharp}}^{\sharp}$ is a compact family with respect to the uniform convergence in $\widehat{\mathbb{C}}$, and

2) every $f \in \mathcal{H}_{\rho^{\sharp}}^{\sharp}$ satisfies the inequality

$$
d^{\sharp}\left(f\left(z_{1}\right), f\left(z_{2}\right)\right) \leq C e^{-\frac{1}{2} \rho^{\sharp}\left(z_{0}, r_{1}, r_{2}\right)}, \quad z_{1}, z_{2} \in B^{\sharp}\left(z_{0}, r_{1}\right),
$$

for $z_{0} \in \widehat{\mathbb{C}}$ and $0<r_{1}<r_{2}<1 / 2 \sqrt{2}$, where $C$ is an absolute constant.

2.3. Proposition. Let $\rho$ be a modulus constraint. Then

1) $\mathcal{H}_{\rho}$ is a compact family with respect to the uniform convergence in $\widehat{\mathbb{C}}$, and

2) for each $R>0$ there is a constant $C=C(R, \rho)>0$ depending only on $R$ and $\rho$ such that every $f \in \mathcal{H}_{\rho}$ satisfies

$$
\left|f\left(z_{1}\right)-f\left(z_{2}\right)\right| \leq C e^{-\rho\left(z_{0}, r_{1}, r_{2}\right)}, \quad z_{1}, z_{2} \in B\left(z_{0}, r_{1}\right),
$$

for $\left|z_{0}\right| \leq R$ and $0<r_{1}<r_{2}<R$. 
In order to prove the propositions, we prepare a few lemmas. For the proof of the first one see [11], where the authors assert that one can take

$$
C_{0}=\pi^{-1} \log 2(1+\sqrt{2})=0.50118 \ldots
$$

below. In fact, it essentially follows from the famous Teichmüller lemma on his extremal ring domain.

2.5. Lemma. There exists a universal constant $C_{0}>0$ with the property that for a ring domain $B$ in $\mathbb{C}$ with $\bmod B>C_{0}$ which separates a point $z_{0}$ from $\infty$ we can choose an annulus $A$ in $B$ of the form $A=A\left(z_{0}, r_{1}, r_{2}\right), r_{1}<r_{2}$, so that $\bmod A \geq \bmod B-C_{0}$.

We need information about the size of components of the complement of a ring domain of sufficiently large modulus. There are several such estimates, and the following form due to Lehto-Virtanen [16, Lemma I.6.1] is probably the best known. Let $B$ be a ring domain whose complement in $\widehat{\mathbb{C}}$ consists of continua $E_{0}$ and $E_{1}$. Then

$$
\min \left\{\operatorname{diam}^{\sharp} E_{0}, \operatorname{diam}^{\sharp} E_{1}\right\} \leq \frac{\pi}{\sqrt{2 \bmod B}} .
$$

However, this bound can be improved when $\bmod B$ is large enough. The following result provides an estimate which has a sharp coefficient of $\bmod B$ in the exponent.

2.6. Lemma. Let $B$ be an arbitrary ring domain in $\widehat{\mathbb{C}}$ and let $E_{0}$ and $E_{1}$ be the components of $\widehat{\mathbb{C}} \backslash B$. Then the inequality

$$
\min \left\{\operatorname{diam}^{\sharp} E_{0}, \operatorname{diam}^{\sharp} E_{1}\right\} \leq C_{1} e^{-\frac{1}{2} \bmod B}
$$

holds where $C_{1}$ is an absolute constant.

Proof. We may assume that $\infty \in E_{1}$. Then we get the desired conclusion by combining Lemma 2.5 with the following elementary but sharp result. In particular, we can use the value $C_{1}=2 e^{C_{0} / 2}=2.56957 \ldots$, where $C_{0}$ is the constant in Lemma 2.5 .

2.7. Lemma. Let $A$ be an annulus in $\widehat{\mathbb{C}}$ whose complement consists of disjoint closed disks $E_{0}$ and $E_{1}$. Then

$$
\min \left\{\operatorname{diam}^{\sharp} E_{0}, \operatorname{diam}^{\sharp} E_{1}\right\} \leq \frac{1}{\cosh \left(\frac{1}{2} \bmod A\right)} .
$$

Equality holds if and only if $\operatorname{diam}^{\sharp} E_{0}=\operatorname{diam}^{\sharp} E_{1}$ and if the spherical centers of $E_{0}$ and $E_{1}$ are antipodal.

Proof. If diam $E_{j}>\operatorname{diam}^{\sharp} E_{1-j}$ for some $j=0,1$, then we can decrease $\operatorname{diam}^{\sharp} E_{j}$ while leaving $\operatorname{diam}^{\sharp} E_{1-j}$ and $\min \left\{\operatorname{diam}^{\sharp} E_{0}, \operatorname{diam}^{\sharp} E_{1}\right\}$ invariant, and the resulting annulus will have larger modulus. Hence we may assume $\operatorname{diam}^{\sharp} E_{0}=\operatorname{diam}^{\sharp} E_{1}$. Performing a suitable isometric Möbius transformation with respect to the spherical metric, we can further assume that $E_{0}$ and $E_{1}$ are symmetric in the imaginary axis and that the center of $E_{0}$ is a positive real number. Let $E_{0} \cap \mathbb{R}=[r, R]$ and $\delta$ be the hyperbolic diameter of $E_{0}$ in the hyperbolic plane $\mathbb{H}=\{z: \operatorname{Re} z>0\}$. Note that

$$
\delta=\int_{r}^{R} \frac{d x}{2 x}=\frac{1}{2} \log \frac{R}{r}=\log t,
$$


where we set $t=\sqrt{R / r}=e^{\delta}>1$. Since $\mathbb{H} \backslash E_{0}$ is Möbius equivalent to the annulus $A(0, \tanh (\delta / 2), 1)$, we can compute the modulus of $A$ as follows:

$$
\bmod A=2 \bmod \left(\mathbb{H} \backslash E_{0}\right)=2 \log \operatorname{coth} \frac{\delta}{2}=2 \log \frac{t+1}{t-1} .
$$

In particular, we have $t=\operatorname{coth}(m / 2)$ where $m=\bmod A / 2$. On the other hand,

$$
\begin{aligned}
\operatorname{diam}^{\sharp} E_{0} & =d^{\sharp}(r, R)=\frac{R-r}{\sqrt{1+R^{2}} \sqrt{1+r^{2}}}=\frac{\left(t^{2}-1\right) r}{\sqrt{1+t^{4} r^{2}} \sqrt{1+r^{2}}} \\
& =\frac{t^{2}-1}{\sqrt{1+t^{4}+r^{-2}+t^{4} r^{2}}} \leq \frac{t^{2}-1}{\sqrt{1+t^{4}+2 t^{2}}}=\frac{t^{2}-1}{t^{2}+1} \\
& =\frac{\operatorname{coth}^{2}(m / 2)-1}{\operatorname{coth}^{2}(m / 2)+1}=\frac{1}{\cosh m},
\end{aligned}
$$

where equality holds if and only if $r t=1$, or equivalently, $r R=1$. The last relation means that the spherical center of $E_{0}$ is 1 and vice versa. In this case, the spherical center of $E_{1}$ is -1 , which is the antipode of 1 . Hence, the last assertion of the lemma follows.

Lemma 2.6 has the sharp coefficient $1 / 2$ in the exponent, however, in the case of ring domains in the finite plane $\mathbb{C}$, the coefficient is no longer best possible. The following estimate has the sharp coefficient 1 in the exponent in this case, although the extra factor $\operatorname{dist}\left(E_{0}, E_{1}\right)$ will come into.

2.8. Lemma. Let $B$ be a ring domain in $\mathbb{C}$ whose complement in $\widehat{\mathbb{C}}$ consists of the bounded component $E_{0}$ and the unbounded component $E_{1}$. Then the inequality

$$
\operatorname{diam} E_{0} \leq C_{3} \operatorname{dist}\left(E_{0}, E_{1}\right) e^{-\bmod B}
$$

holds provided that $\bmod B>C_{2}$, where $C_{2}$ and $C_{3}$ are positive absolute constants.

Proof. We may assume that $\operatorname{dist}\left(E_{0}, E_{1}\right)=1,0 \in E_{0}$ and $1 \in E_{1}$. Let $a \neq 0$ be an arbitrary point in $E_{0}$. Then, by Teichmüller's modulus theorem (see [16]), we have

$$
\bmod B \leq 2 \mu\left(\sqrt{\frac{|a|}{1+|a|}}\right),
$$

where $\mu(r)$ denotes the modulus of the Grötszch ring $B(0,1) \backslash[0, r]$. Using the well-known estimate $\mu(r)<\log (4 / r)$, we obtain

$$
|a| \leq \frac{16}{e^{\bmod B}-16} \leq 32 e^{-\bmod B}
$$

if $\bmod B>5 \log 2$. Hence, $\operatorname{diam} E_{0} \leq 64 e^{-\bmod B}$ whenever $\bmod B>5 \log 2 . \operatorname{In}$ particular, the assertion holds for $C_{2}=5 \log 2$ and $C_{3}=64$.

Proof of Proposition [2.1. Observe first that $d^{\sharp}(0,1)=d^{\sharp}(1, \infty)=1 / \sqrt{2}$ and that $d^{\sharp}(0, \infty)=1$. In particular, for $r_{2}<1 / 2 \sqrt{2}$, the disk $B=B^{\sharp}\left(z_{0}, r_{2}\right)$ cannot contain more than one of the three fixed points $0,1, \infty$. Therefore, the component $E_{1}=$ $\widehat{\mathbb{C}} \backslash f(B)$ of the complement of $f\left(A^{\sharp}\left(z_{0}, r_{1}, r_{2}\right)\right)$ has spherical diameter at least $1 / \sqrt{2}$. Meanwhile, the other component $E_{0}=f\left(\bar{B}^{\sharp}\left(z_{0}, r_{1}\right)\right)$ has spherical diameter at most 1 . Consequently, $\operatorname{diam}^{\sharp} E_{0} \leq \sqrt{2} \min \left\{\operatorname{diam}^{\sharp} E_{0}, \operatorname{diam}^{\sharp} E_{1}\right\}$. Now inequality (2.2) follows from Lemma [2.6 with $C=\sqrt{2} C_{1}$, where $C_{1}$ is the constant appearing 
in the lemma. This inequality in turn implies the equicontinuity of $\mathcal{H}_{\rho^{\sharp}}^{\sharp}$. Since $\widehat{\mathbb{C}}$ is compact, then by the Arzelà-Ascoli theorem, the family $\mathcal{H}_{\rho^{\sharp}}^{\sharp}$ is normal.

Let $f$ be the uniform limit of a sequence $f_{n}$ in $\mathcal{H}_{\rho^{\sharp}}^{\sharp}$. We show that $f$ is a member of $\mathcal{H}_{\rho^{\sharp}}^{\sharp}$. Since the mapping degree is preserved under uniform convergence, $f$ has degree 1 , in particular, $f: \widehat{\mathbb{C}} \rightarrow \widehat{\mathbb{C}}$ is a surjective continuous map. We now consider the open set

$$
V=\{z \in \widehat{\mathbb{C}} ; f \text { is locally constant at } z\} .
$$

First we show the following

Claim. If $z_{0} \in \widehat{\mathbb{C}} \backslash V$, then $f(z) \neq f\left(z_{0}\right)$ for $z \in \widehat{\mathbb{C}} \backslash\left\{z_{0}\right\}$.

By permuting the roles of $0,1, \infty$ if necessary, we may assume that $z_{0} \neq \infty$ and that $f\left(z_{0}\right) \neq \infty$. Pick a point $w_{0} \neq z_{0}$. We will show that $f\left(w_{0}\right) \neq f\left(z_{0}\right)$. Choose a small positive number $R$ so that $R<\min \left\{d^{\sharp}\left(z_{0}, w_{0}\right), d^{\sharp}\left(z_{0}, \infty\right)\right\}$. Then, by the definition of $\mathcal{H}_{\rho^{\sharp}}^{\sharp}, \inf _{n} \bmod \left(f_{n}\left(A^{\sharp}\left(z_{0}, \delta, R\right)\right)\right)>C_{0}$ for sufficiently small $\delta>0$, where $C_{0}$ is the constant in Lemma 2.5. By virtue of Lemma 2.5, we can find an annulus $A_{n}$ of the form $A\left(f_{n}\left(z_{0}\right), r_{n}, r_{n}^{\prime}\right), r_{n}<r_{n}^{\prime}$, in $f_{n}\left(A^{\sharp}\left(z_{0}, \delta, R\right)\right)$ for $n$ large enough.

Since $f$ is not locally constant at $z_{0}$, there exists a point $z_{1}$ in the disk $d^{\sharp}\left(z, z_{0}\right)<$ $\delta$ with $f\left(z_{0}\right) \neq f\left(z_{1}\right)$. The annulus $A_{n}$ separates $f_{n}\left(z_{0}\right), f_{n}\left(z_{1}\right)$ from $f_{n}\left(w_{0}\right), \infty$, so we obtain $\left|f_{n}\left(z_{1}\right)-f_{n}\left(z_{0}\right)\right| \leq r_{n}$ and $r_{n}^{\prime} \leq\left|f_{n}\left(w_{0}\right)-f_{n}\left(z_{0}\right)\right|$. In particular, $\left|f_{n}\left(z_{1}\right)-f_{n}\left(z_{0}\right)\right| \leq\left|f_{n}\left(w_{0}\right)-f_{n}\left(z_{0}\right)\right|$ for $n$ large enough. Letting $n \rightarrow \infty$, we obtain $0<\left|f\left(z_{1}\right)-f\left(z_{0}\right)\right| \leq\left|f\left(w_{0}\right)-f\left(z_{0}\right)\right|$, and hence $f\left(w_{0}\right) \neq f\left(z_{0}\right)$.

We next show that $V$ is empty. Suppose that $V$ has a non-empty component $V_{0}$. Then $f$ takes a constant value, say $b$, in $V_{0}$. Because $V_{0} \neq \widehat{\mathbb{C}}$, there is a point $z_{0}$ in $\partial V_{0}$. By continuity, we have $f\left(z_{0}\right)=b$. On the other hand, from the above Claim it follows that $f(z) \neq f\left(z_{0}\right)=b$ for any point $z$ other than $z_{0}$, which contradicts the fact that $f=b$ in $V_{0}$. Thus we conclude that $V$ is empty, namely, $f$ is not locally constant at any point.

By using the Claim again, we obtain $f\left(z_{1}\right) \neq f\left(z_{2}\right)$ if $z_{1} \neq z_{2}$. Thus, the injectivity of $f$ follows. Finally, the continuity of moduli of ring domains with respect to the Hausdorff convergence (see [9]) implies that $f \in \mathcal{H}_{\rho^{\sharp}}^{\sharp}$.

Proof of Proposition 2.3. It is easy to see that there is a spherical modulus constraint $\rho^{\sharp}$ such that $\mathcal{H}_{\rho} \subset \mathcal{H}_{\rho^{\sharp}}^{\sharp}$. From Proposition 2.1, the compactness of $\mathcal{H}_{\rho}$ now follows. We next give an estimate of $\operatorname{dist}\left(E_{0}, E_{1}\right)$, where $E_{0}$ and $E_{1}$ are the bounded and unbounded components of $\widehat{\mathbb{C}} \backslash f(A), A=A\left(z_{0}, r_{1}, r_{2}\right)$, respectively. Since $\mathcal{H}_{\rho}$ is compact, $S=\sup \left\{|g(z)|: g \in \mathcal{H}_{\rho},|z| \leq 2 R\right\}<+\infty$. Now it is clear that $\operatorname{dist}\left(E_{0}, E_{1}\right) \leq \operatorname{diam} f\left(\partial B\left(z_{0}, r_{2}\right)\right) \leq 2 S$. Set $m=\bmod f(A)$. If $m>C_{2}$, we obtain $\operatorname{diam} E_{0} \leq 2 S C_{3} e^{-m}$ by Lemma 2.8. Otherwise, we have $\operatorname{diam} E_{0} \leq 2 S \leq 2 S e^{C_{2}} e^{-m}$. Hence, the proof is complete.

Let $\mu$ be a Beltrami coefficient defined in $\mathbb{C}$ with $\|\mu\|_{\infty} \leq 1$. In order to apply Proposition 2.3 to the study of the Beltrami equation, we first introduce a standard approximation procedure. For $n=1,2, \ldots$, we set

$$
\mu_{n}(z)=\mu(z), \quad \text { if }|\mu(z)| \leq 1-1 / n,
$$

and $\mu_{n}(z)=0$ otherwise, and denote by $f_{n}$ the sequence of quasiconformal automorphisms of the extended complex plane preserving 0,1 and $\infty$, and having $\mu_{n}$ 
as its complex dilatation. The existence of such $f_{n}$ is guaranteed by the measurable Riemann mapping theorem. We will call such $f_{n}$ the canonical approximating sequence for $\mu$. The topological structure of the family $\left\{f_{n}\right\}$ with respect to the uniform convergence under some additional assumptions on $\mu$ will be described in Theorem 2.15.

A function $H:[0,+\infty) \rightarrow \mathbb{R}$ is called a dominating factor if the following conditions are satisfied:

1. $H(x)$ is continuous and strictly increasing in $\left[x_{0},+\infty\right)$ and $H(x)=H\left(x_{0}\right)$ for $x \in\left[0, x_{0}\right]$ for some $x_{0} \geq 0$, and

2. the function $e^{H(x)}$ is convex in $x \in[0,+\infty)$.

The convexity of $e^{H}$ implies that $H(x) \rightarrow+\infty$ as $x \rightarrow+\infty$. In the sequel, the inverse $H^{-1}$ of $H$ will mean the inverse of the homeomorphism $H:\left[x_{0},+\infty\right) \rightarrow$ $[H(0),+\infty)$.

A dominating factor $H$ is said to be of divergence type if

$$
\int_{1}^{+\infty} \frac{H(x) d x}{x^{2}}=+\infty
$$

and otherwise $H$ is said to be of convergence type. Denote by $\mathcal{D}$ the set of all dominating factors of divergence type. Note that $H(\eta x)$ and $\eta H(x)$ are dominating factors of the same type as $H(x)$ if $H(x)$ is a dominating factor and if $\eta$ is a positive constant.

2.11. Lemma. Let $H$ be a dominating factor. Then $H$ is of divergence type if and only if

$$
\int_{t_{1}}^{+\infty} \frac{d t}{H^{-1}(t)}=+\infty
$$

for a sufficiently large number $t_{1}$.

Proof. By the change of variables $t=H(x)$ and integration by parts, we see

$$
\int_{t_{1}}^{t_{2}} \frac{d t}{H^{-1}(t)}=\int_{x_{1}}^{x_{2}} \frac{d H(x)}{x}=\frac{H\left(x_{2}\right)}{x_{2}}-\frac{H\left(x_{1}\right)}{x_{1}}+\int_{x_{1}}^{x_{2}} \frac{H(x) d x}{x^{2}},
$$

where $t_{j}=H\left(x_{j}\right)$ for $j=1,2$. Now the "only if" part follows because $H(x) / x$ is positive for large $x$. In order to show the "if" part, assume that (2.10) fails. Then, in view of the above identity, (2.12) would imply that $H(x) / x \rightarrow \infty$ if $x \rightarrow \infty$. In particular, $H(x) / x>C>0$ holds for sufficiently large $x$, where $C$ is a positive constant. Hence, for $x_{1}$ large enough, we obtain $\int_{x_{1}}^{x_{2}} H(x) d x / x^{2}>\int_{x_{1}}^{x_{2}} C d x / x=$ $C \log \left(x_{2} / x_{1}\right) \rightarrow \infty$ as $x_{2} \rightarrow \infty$, a contradiction. Thus, (2.10) follows from (2.12).

Let $\mu \in L^{\infty}(\Omega)$ be a Beltrami coefficient with $|\mu|<1$ a.e. in an open subset $\Omega$ of $\widehat{\mathbb{C}}$. We define the angular dilatation $D_{\mu, z_{0}}$ of $\mu$ at $z_{0} \in \widehat{\mathbb{C}}$ as

$$
D_{\mu, z_{0}}(z)=\frac{\left|1-\mu(z) \frac{\bar{z}-\bar{z}_{0}}{z-z_{0}}\right|^{2}}{1-|\mu(z)|^{2}}
$$

in the case when $z_{0}$ is finite, and as $D_{\mu, \infty}(z)=D_{\mu, 0}(z)$ in the case when $z_{0}=\infty$. Then $D_{\mu, z_{0}}$ is a measurable function in $\Omega$ and satisfies the inequality $1 / K_{\mu}(z) \leq$ $D_{\mu, z_{0}}(z) \leq K_{\mu}(z)$ a.e. for each $z_{0} \in \widehat{\mathbb{C}}$. Note that $D_{\mu, 0}(z)=K_{\mu}(z)$ holds a.e. if and only if $\mu(z)$ has the form $-\rho(z) z / \bar{z}$ for a non-negative measurable function $\rho$. The 
name of $D_{\mu, z_{0}}$ comes from the following important relation: if $f$ is $\mu$-conformal in $\Omega$ and if we write $z=z_{0}+r e^{i \theta}$, then

$$
\left|\frac{\partial f}{\partial \theta}(z)\right|^{2}=r^{2} D_{\mu, z_{0}}(z) J_{f}(z)
$$

holds for almost all $z \in \Omega$, where $J_{f}$ is the Jacobian of $f$. The quantity $D_{-\mu, z_{0}}$ is called the radial dilatation of $\mu$ at $z_{0}$ because it satisfies

$$
\left|\frac{\partial f}{\partial r}(z)\right|^{2}=D_{-\mu, z_{0}}(z) J_{f}(z)
$$

We call $D_{\mu, z_{0}}$ and $D_{-\mu, z_{0}}$ directional dilatations.

2.15. Theorem. Let $\mu$ be a Beltrami coefficient in $\mathbb{C}$. Assume that for each $z_{0} \in \widehat{\mathbb{C}}$ one of the following conditions holds for some positive constants $M=M\left(z_{0}\right)$ and $r_{0}=r_{0}\left(z_{0}\right)$ :

1) $D_{\mu, z_{0}}(z) \leq M$ a.e. in $B\left(z_{0}, r_{0}\right)$;

2) there is a dominating factor $H=H_{z_{0}}$ of divergence type such that

$$
\int_{B\left(z_{0}, r_{0}\right)} e^{H\left(D_{\mu, z_{0}}(z)\right)} d \mathcal{A}(z) \leq M
$$

holds when $z_{0} \in \mathbb{C}$, while the above condition is replaced by

$$
\int_{B\left(\infty, r_{0}\right)} e^{H\left(D_{\mu, 0}(z)\right)} \frac{d \mathcal{A}(z)}{|z|^{4}} \leq M
$$

when $z_{0}=\infty$.

Then the canonical approximating sequence $f_{n}$ for $\mu$ forms a normal family with respect to the uniform convergence in $\widehat{\mathbb{C}}$ and every limit function $f$ of this sequence is a self-homeomorphism of $\widehat{\mathbb{C}}$. Moreover, $f$ admits the following modulus of continuity estimates according to cases 1 ) or 2 ) at each point $z_{0}$ with $\left|z_{0}\right| \leq R_{0}$, where $R_{0}$ is an arbitrary positive number:

$$
\left|f(z)-f\left(z_{0}\right)\right| \leq C\left|z-z_{0}\right|^{1 / M}
$$

or

$$
\left|f(z)-f\left(z_{0}\right)\right| \leq C \exp \left\{-\int_{1+c}^{2 m+c} \frac{d t}{2 H^{-1}(t)}\right\},
$$

respectively, for $\left|z-z_{0}\right|<r_{1}$ and $0<r_{1} \leq \min \left\{r_{0}, R_{0}\right\}$, where $m=\log \left(r_{1} /\left|z-z_{0}\right|\right)$, $c=\log \left(M / \pi r_{1}^{2}\right)$ and $C$ is a constant depending only on $\mu$ and $R_{0}$.

2.18. Remark. Note that Theorem 2.15 does not say anything about the $\mu$-conformal mapping; it discusses the behavior of the limit mapping only. However, the following conclusions can be made. Let $\operatorname{Sing}(\mu)$ be the singular set of a Beltrami coefficient $\mu$, i.e.,

$$
\operatorname{Sing}(\mu)=\left\{z_{0} \in \widehat{\mathbb{C}}: \operatorname{ess} \limsup _{z \rightarrow z_{0}}|\mu(z)|=1\right\} .
$$

Note that $\operatorname{Sing}(\mu)$ is a compact set in $\widehat{\mathbb{C}}$. Note also that $\operatorname{Sing}(\mu)$ can have positive area although $|\mu|$ is always assumed to be less than 1 almost everywhere. By definition, the open set $\Omega=\widehat{\mathbb{C}} \backslash \operatorname{Sing}(\mu)$ can be exhausted by a sequence of open subsets $\Omega_{n}, n=1,2, \ldots$, in such a way that $|\mu| \leq 1-1 / n$ a.e. in $\Omega_{n}$. Since $\mu_{k}=\mu$ a.e. in $\Omega_{n}$ for all $k \geq n$, the limit $f$ is $\mu$-conformal in $\Omega_{n}$ for each $n$. 
Therefore, $f$ is locally quasiconformal in $\Omega$, in particular, $f \in W_{\text {loc }}^{1,2}(\Omega)$, the complex dilatation of $f$ agrees with the given $\mu$ a.e. in $\Omega$, and $\left.f\right|_{\Omega}$ is unique up to the post-composition by a conformal map. Further discussions on the existence of $\mu$ conformal homeomorphisms and their regularity will be made in the next section.

The proof of the theorem is based on Proposition 2.3 and an estimate on the moduli of ring domains under homeomorphisms in the Sobolev space $W_{\text {loc }}^{1,1}$ stated in the lemmas below.

The first result can essentially be found in 2] and [20]. We give it under somewhat weaker assumptions.

2.19. Lemma. Let $\mu$ be a Beltrami coefficient on a domain $\Omega$ in $\mathbb{C}$ and let $f: \Omega \rightarrow$ $\mathbb{C}$ be a $\mu$-conformal embedding. Suppose that $D_{\mu, z_{0}}(z)$ is locally integrable in the annulus $A=A\left(z_{0}, r_{1}, r_{2}\right) \subset \Omega$. Then

$$
\bmod f(A) \geq \int_{r_{1}}^{r_{2}} \frac{d r}{r \psi_{\mu}\left(r, z_{0}\right)},
$$

where

$$
\psi_{\mu}\left(r, z_{0}\right)=\frac{1}{2 \pi} \int_{0}^{2 \pi} D_{\mu, z_{0}}\left(z_{0}+r e^{i \theta}\right) d \theta .
$$

Proof. We may assume that $z_{0}=0$ and $\Omega=A=A(0,1, R)$. We may further assume that $A^{\prime}=f(A)=A\left(0,1, R^{\prime}\right)$. Let $\nu$ be the positive Borel measure on $A$ defined by $\nu(E)=\mathcal{A}(f(E))$. By a usual argument (see, e.g., 1] or [16]), we obtain $J_{f} d \mathcal{A} \leq d \nu$ on $A$ in the sense of measure, where $J_{f}=\left|f_{z}\right|^{2}-\left|f_{\bar{z}}\right|^{2}$. In particular, $J_{f} \in L_{\mathrm{loc}}^{1}(A)$.

Denote by $\gamma_{r}$ the circle $|z|=r$. Then the assumption $f \in W_{\text {loc }}^{1,1}(A)$ together with the Gehring-Lehto theorem (see [16]) implies that, for almost all $r \in(1, R), f$ is absolutely continuous on $\gamma_{r}$ and totally differentiable at every point in $\gamma_{r}$ except for a set of linear measure 0. By Fubini's theorem, we observe that $D_{\mu}$ and $J_{f}$ are integrable on $\gamma_{r}$ for almost all $r \in(1, R)$. For such an $r$, we have

$$
2 \pi \leq \int_{\gamma_{r}}|d \arg f| \leq \int_{\gamma_{r}} \frac{|d f(z)|}{|f(z)|}=\int_{0}^{2 \pi} \frac{\left|f_{\theta}\left(r e^{i \theta}\right)\right|}{\left|f\left(r e^{i \theta}\right)\right|} d \theta .
$$

We use Schwarz's inequality and (2.14) to obtain

$$
(2 \pi)^{2} \leq r^{2} \int_{0}^{2 \pi} D_{\mu}\left(r e^{i \theta}\right) d \theta \int_{0}^{2 \pi} \frac{J_{f}}{|f|^{2}}\left(r e^{i \theta}\right) d \theta
$$

and hence

$$
\frac{2 \pi}{r \psi_{\mu}(r)} \leq r \int_{0}^{2 \pi} \frac{J_{f}}{|f|^{2}}\left(r e^{i \theta}\right) d \theta
$$

for almost all $r \in(1, R)$, where $\psi_{\mu}(r)=\psi_{\mu}(r, 0)$. Integrating both sides with respect to $r$ from 1 to $R$, we obtain

$$
\begin{aligned}
& 2 \pi \int_{1}^{R} \frac{d r}{r \psi_{\mu}(r)} \leq \int_{1}^{R} \int_{0}^{2 \pi} \frac{J_{f}}{|f|^{2}} r d \theta d r=\int_{A} \frac{J_{f} d \mathcal{A}}{|f|^{2}} \\
& \leq \int_{A} \frac{d \nu}{|f|^{2}}=\int_{A^{\prime}} \frac{d \mathcal{A}(w)}{|w|^{2}}=2 \pi \log R^{\prime}=2 \pi \bmod A^{\prime}
\end{aligned}
$$

and thus arrive at the required inequality (2.20). 
The following auxiliary result may be of independent interest. The basic idea is due to Brakalova-Jenkins [6]; see also [18, p. 51].

2.22. Lemma. Let $f$ be a $\mu$-conformal embedding of $A=A\left(z_{0}, r_{0} e^{-m}, r_{0}\right)$ into $\mathbb{C}$. Suppose that a dominating factor $H$ satisfies

$$
\begin{gathered}
\int_{A} e^{H\left(D_{\mu, z_{0}}(z)\right)} d \mathcal{A}(z) \leq M, \quad \text { if } z_{0} \in \mathbb{C}, \text { and } \\
\int_{A} e^{H\left(D_{\mu, 0}(z)\right)} \frac{d \mathcal{A}(z)}{|z|^{4}} \leq M, \quad \text { if } z_{0}=\infty .
\end{gathered}
$$

Then we have

$$
\bmod f\left(A\left(z_{0}, r_{0} e^{-m}, r_{0}\right)\right) \geq \int_{1 / 2}^{m} \frac{d t}{H^{-1}\left(2 t+\log \left(M / \pi r_{0}^{2}\right)\right)} .
$$

Proof. Let first $z_{0} \neq \infty$. Setting

$$
h(r)=\frac{r^{2}}{2 \pi} \int_{0}^{2 \pi} e^{H\left(D_{\mu, z_{0}}\left(z_{0}+r e^{i \theta}\right)\right)} d \theta,
$$

we rewrite inequality (2.23) in the form

$$
2 \pi \int_{r_{0} e^{-m}}^{r_{0}} h(r) \frac{d r}{r}=2 \pi \int_{0}^{m} h\left(r_{0} e^{-t}\right) d t \leq M .
$$

By Chebyshev's inequality, the set $T=\left\{t \in(0, m): h\left(r_{0} e^{-t}\right)>L\right\}$ has the length

$$
|T|=\int_{T} d t \leq \frac{M}{2 \pi L}
$$

Since $e^{H}$ is a convex function, Jensen's inequality yields $e^{H(\psi(r))} \leq h(r) / r^{2}$, where $\psi(r)=\psi_{\mu}\left(r, z_{0}\right)$. This implies the inequality $\psi\left(r_{0} e^{-t}\right) \leq H^{-1}\left(2 t+\log \left(L / r_{0}^{2}\right)\right)$ for $t \in(0, m) \backslash T$. Lemma 2.19 now yields

$$
\begin{aligned}
\bmod f(A) & \geq \int_{0}^{m} \frac{d t}{\psi\left(r_{0} e^{-t}\right)} \geq \int_{(0, m) \backslash T} \frac{d t}{H^{-1}\left(2 t+\log \left(L / r_{0}^{2}\right)\right)} \\
& \geq \int_{|T|}^{m} \frac{d t}{H^{-1}\left(2 t+\log \left(L / r_{0}^{2}\right)\right)} \geq \int_{M / 2 \pi L}^{m} \frac{d t}{H^{-1}\left(2 t+\log \left(L / r_{0}^{2}\right)\right)} .
\end{aligned}
$$

Finally, letting $L=M / \pi$, we obtain (2.24).

The remaining case is that $z_{0}=\infty$. Let $\varphi(z)=1 / z$ be the inversion. Let $\hat{\mu}$ be the complex dilatation of the map $g=\varphi \circ f \circ \varphi$. Then $D_{\hat{\mu}, 0}(z)=D_{\mu, 0}(1 / z)$. Now the required inequality immediately follows from the previous one.

Proof of Theorem [2.15, Let $f_{n}$ be the canonical approximating sequence for $\mu$. First suppose that $\mu$ satisfies assumption 2$)$ at $z_{0} \in \mathbb{C}$. Since

$$
H \circ D_{\mu_{n}, z_{0}}(z) \leq \max \left\{H \circ D_{\mu, z_{0}}(z), H(1)\right\}
$$

for the dominating factor $H$, the sequence

$$
M_{n}=\int_{B\left(z_{0}, r_{0}\right)} e^{H\left(D_{\mu_{n}, z_{0}}(z)\right)} d \mathcal{A}(z)
$$

satisfies

$$
\lim _{n \rightarrow \infty} M_{n}=\int_{B\left(z_{0}, r_{0}\right)} e^{H\left(D_{\mu, z_{0}}(z)\right)} d \mathcal{A}(z) \leq M=M\left(z_{0}\right)
$$


by Lebesgue's dominating convergence theorem. Set $\widehat{M}_{n}=\sup \left\{M_{k} ; k \geq n\right\}$. We now see from Lemma 2.22 that

$$
\bmod f_{n}\left(A\left(z_{0}, r e^{-m}, r\right)\right) \geq \int_{1 / 2}^{m} \frac{d t}{H^{-1}\left(2 t+\log \left(\widehat{M}_{n} / \pi r^{2}\right)\right)}=: \rho_{n}\left(z_{0}, r e^{-m}, r\right)
$$

for $0<r \leq r_{0}$ and $m>1 / 2$. Then, by virtue of Lemma [2.11] $\rho_{n}\left(z_{0}, r e^{-m}, r\right) \rightarrow \infty$ as $m \rightarrow+\infty$. For $z_{0}=\infty$ the same conclusion follows.

In the case when $\mu$ satisfies assumption 1$)$, the situation is simpler. Since $\psi_{\mu}\left(r, z_{0}\right) \leq M$ for a.e. $0<r \leq r_{0}$, by Lemma 2.19, we obtain $\bmod f_{n}\left(A\left(z_{0}, r e^{-m}, r\right)\right)$ $\geq m / M$ for $0<r \leq r_{0}$ and $m>0$, so we just set $\rho_{n}\left(z_{0}, r e^{-m}, r\right)=m / M$ in this case. For the other points $\left(z_{0}, r, R\right)$, we simply set $\rho_{n}\left(z_{0}, r, R\right)=0$.

The sequence $f_{k}, k \geq n$, then belongs to the class $\mathcal{H}_{\rho_{n}}$ for each $n$. By Proposition 2.3, the limit map $f$ of any convergent subsequence of $f_{k}$ is homeomorphic. We show the estimates of modulus of continuity of $f$. Since case 1 ) is easier to treat, we consider only case 2). Noting that $f \in \mathcal{H}_{\rho_{n}}$ for each $n$, we obtain $f \in \mathcal{H}_{\rho_{\infty}}$, where $\rho_{\infty}=\lim _{n \rightarrow \infty} \hat{\rho}_{n}$. By Proposition 2.3, we now have

$$
\left|f(z)-f\left(z_{0}\right)\right| \leq C \exp \left(-\rho_{\infty}\left(z_{0}, r_{1} e^{-m}, r_{1}\right)\right)
$$

for $\left|z-z_{0}\right| \leq r_{1} e^{-m},\left|z_{0}\right| \leq R_{0}$ and $r_{1} \leq \min \left\{r_{0}\left(z_{0}\right), R_{0}\right\}$, where $C$ is a constant depending only on $\mu$ and $R_{0}$. We now obtain 2.17 by

$$
\rho_{\infty}\left(z_{0}, r_{1} e^{-m}, r_{1}\right) \geq \int_{1 / 2}^{m} \frac{d t}{H^{-1}\left(2 t+\log \left(M / \pi r_{1}^{2}\right)\right)}=\int_{1+c}^{2 m+c} \frac{d t}{2 H^{-1}(t)},
$$

where $c=\log \left(M / \pi r_{1}^{2}\right)$.

\section{Existence THEOREMS}

We first recall some regularity results (see, e.g., [6], Lemmas $4-6$ and Proposition 10, and also [19], 18]) on the degenerate Beltrami equation and then use them and Theorem 2.15 to study the existence problem of the solutions to the aforementioned equation.

3.1. Proposition. Let $\mu$ be a Beltrami coefficient in $\mathbb{C}$ and suppose that a homeomorphism $f: \widehat{\mathbb{C}} \rightarrow \widehat{\mathbb{C}}$ is a uniform limit of the canonical approximating sequence $f_{n}$ for $\mu$. If $K_{\mu} \in L_{\mathrm{loc}}^{p}(\mathbb{C})$ for some $p>1$, then $f \in W_{\mathrm{loc}}^{1, q}(\mathbb{C}), q=2 p /(1+p)$, and $f$ satisfies the Beltrami equation with $\mu$. Moreover, $f^{-1} \in W_{\mathrm{loc}}^{1,2}(\mathbb{C})$.

3.2. Remark. It is shown in [6, Lemma 3] that $K_{\mu} \in L_{\text {loc }}^{1}(\mathbb{C})$ implies the ACL property of $f$.

3.3. Remark. The Sobolev embedding theorem for spheres 17] or Gehring's oscillation inequality (see, e.g., [12, Lemma 5.2]) implies that if $f: \mathbb{C} \rightarrow \mathbb{C}$ is a $\mu$-conformal homeomorphism with $K_{\mu} \in L_{\text {loc }}^{1}(\mathbb{C})$, then for every compact set $E$ in $\mathbb{C}$ there exist constants $C$ and $a$ such that

$$
\left|f\left(z_{1}\right)-f\left(z_{2}\right)\right| \geq C e^{-a /\left|z_{1}-z_{2}\right|^{2}}
$$

for $z_{1}, z_{2} \in E$ with $z_{1} \neq z_{2}$. The same inequality is also obtained in [23, p. 75] as a consequence of the Length-Area principle. 
Proposition 2.1 together with Theorem 2.15 yields the following statement.

3.5. Theorem. Suppose that $\mu$ is a Beltrami coefficient in $\mathbb{C}$ such that:

a) $K_{\mu} \in L_{\text {loc }}^{p}(\mathbb{C})$ for some $p>1$; and

b) for each point $z_{0} \in \widehat{\mathbb{C}}$ there exist positive constants $M=M\left(z_{0}\right)$ and $r_{0}=$ $r_{0}\left(z_{0}\right)$ so that either 1$) D_{\mu, z_{0}} \leq M$ a.e. in $B\left(z_{0}, r_{0}\right)$ or 2$)$

$$
\begin{aligned}
& \int_{B\left(z_{0}, r_{0}\right)} e^{H\left(D_{\mu, z_{0}}(z)\right)} d \mathcal{A}(z) \leq M, \quad \text { when } z_{0} \in \mathbb{C}, \text { and } \\
& \int_{B\left(\infty, r_{0}\right)} e^{H\left(D_{\mu, 0}(z)\right)} \frac{d \mathcal{A}(z)}{|z|^{4}} \leq M, \quad \text { when } z_{0}=\infty
\end{aligned}
$$

holds for some dominating factor $H=H_{z_{0}}$ of divergence type.

Then there exists a normalized homeomorphic solution $f: \widehat{\mathbb{C}} \rightarrow \widehat{\mathbb{C}}$ of (1.1) such that $f \in W_{\mathrm{loc}}^{1, q}(\mathbb{C}), q=2 p /(1+p)$, and $f^{-1} \in W_{\mathrm{loc}}^{1,2}(\mathbb{C})$. This homeomorphism admits the modulus of continuity estimate either $\left|f(z)-f\left(z_{0}\right)\right| \leq C\left|z-z_{0}\right|^{1 / M}$ or

$$
\left|f(z)-f\left(z_{0}\right)\right| \leq C \exp \left\{-\frac{1}{2} \int_{1+c}^{2 m+c} \frac{d t}{H_{z_{0}}^{-1}(t)}\right\},
$$

respectively according to case 1) or 2), for $\left|z-z_{0}\right|<r_{1},\left|z_{0}\right| \leq R_{0}$ and $r_{1} \leq$ $\min \left\{r_{0}\left(z_{0}\right), R_{0}\right\}$, where $m=\log \left(r_{1} /\left|z-z_{0}\right|\right), c=\log \left(M\left(z_{0}\right) / \pi r_{1}^{2}\right), R_{0}>0$ is a fixed number and $C$ is a constant depending only on $\mu$ and $R_{0}$.

Proof. Let $f_{n}$ be the canonical approximating sequence corresponding to the Beltrami coefficient $\mu$. From Theorem 2.15 we see that $f_{n}$ forms a pre-compact family with respect to the uniform convergence in $\widehat{\mathbb{C}}$ and every limit function $f$ of this family is a self-homeomorphism of $\widehat{\mathbb{C}}$. Passing to a subsequence, we may assume that $f_{n} \rightarrow f$ uniformly in $\widehat{\mathbb{C}}$ and $\mu_{n} \rightarrow \mu$ a.e. as $n \rightarrow \infty$. Since $K_{\mu} \in L_{\text {loc }}^{p}$ ( $\mathbb{C}$ ) for some $p>1$, we see by Proposition 3.1 that $f \in W_{\mathrm{loc}}^{1, q}(\mathbb{C}), q=2 p /(1+p)$ and $f$ satisfies (1.1). Moreover, $f_{n}^{-1} \rightarrow f^{-1}$ uniformly in $\widehat{\mathbb{C}}$ as $n \rightarrow \infty$ and $f^{-1} \in W_{\text {loc }}^{1,2}(\mathbb{C})$. The modulus of continuity estimate in (3.7) follows from Theorem 2.15.

3.8. Remark. Assumption b) in Theorem 3.5 implies $D_{\mu, z_{0}} \in L^{p}\left(B\left(z_{0}, r_{0}\right)\right)$ for all $p>1$. Assumption a), however, cannot be dropped in order to have the regularity condition $f \in W_{\text {loc }}^{1,1}(\mathbb{C})$. Indeed, for $\mu(z)=\left(1+i|z|^{2}\right)^{-1} z / \bar{z},|z|<1$, and $\mu(z)=$ $0,|z|>1$, the normalized $\mu$-conformal homeomorphism $f: \widehat{\mathbb{C}} \rightarrow \widehat{\mathbb{C}}$ has the form $f(z)=z e^{i\left(1 /|z|^{2}-1\right)},|z|<1$, and $f(z)=z,|z| \geq 1$. A simple calculation shows that $D_{\mu, 0}(z)=1$ a.e. in $\mathbb{C}$, and therefore $D_{\mu, z_{0}}$ is bounded near $z_{0}$ for each $z_{0} \in \widehat{\mathbb{C}}$. Hence, assumption b) is fulfilled, but $\left|f_{\bar{z}}\right|=1 /|z|^{2}$ is not locally integrable near the origin, and thus $f \notin W_{\text {loc }}^{1,1}(\mathbb{C})$. Note that $K_{\mu}(z) \geq 1 /|z|^{4}$ and hence $K_{\mu} \notin L_{\text {loc }}^{1}(\mathbb{C})$.

Note also that assumption a) can be stated in terms of the radial dilatation of $\mu$. In fact, we can replace it by the condition that $D_{-\mu, z_{0}}$ belongs to $L^{p}\left(B\left(z_{0}, r_{0}\right)\right)$ for each finite $z_{0}$, where $p>1$ is a fixed number. This follows from the double inequality

$$
\frac{K_{\mu}}{2} \leq D_{\mu, z_{0}}+D_{-\mu, z_{0}}=\frac{1+|\mu|^{2}}{1-|\mu|^{2}} \leq K_{\mu}
$$


Specifying appropriate dominating factors $H(x)$ we obtain more concrete consequences from Theorem 3.5. Typical choices for $H(x)$ are $\eta x$ and $\eta x /\left(1+\log ^{+} x\right)$ for a positive constant $\eta$. The integrability condition (3.6) now reduces to

$$
\int_{B\left(z_{0}, r_{0}\right)} e^{\eta D_{\mu, z_{0}}(z)} d \mathcal{A}^{\sharp}(z)<+\infty
$$

and to the local subexponential integrability condition

$$
\int_{B\left(z_{0}, r_{0}\right)} \exp \left\{\frac{\eta D_{\mu, z_{0}}(z)}{1+\log ^{+} D_{\mu, z_{0}}(z)}\right\} d \mathcal{A}^{\sharp}(z)<+\infty,
$$

respectively. More examples will be given in Section 5 .

The following result can be viewed as an extended version of the corresponding existence theorems from [6] and [12].

3.11. Theorem. Let $H$ be a dominating factor of divergence type. Suppose that a Beltrami coefficient $\mu$ on $\mathbb{C}$ with $\|\mu\|_{\infty} \leq 1$ satisfies

$$
\int_{\mathbb{C}} e^{H\left(K_{\mu}(z)\right)} d \mathcal{A}^{\sharp}(z)<+\infty,
$$

where $d \mathcal{A}^{\sharp}(z)=\left(1+|z|^{2}\right)^{-2} d \mathcal{A}(z)$ denotes the spherical area element. Then there exists a normalized homeomorphic solution $f$ to the Beltrami equation such that $f \in W_{\text {loc }}^{1, q}(\mathbb{C})$ for every $1 \leq q<2$ and that $f^{-1} \in W_{\text {loc }}^{1,2}(\mathbb{C})$.

Proof. Since $H$ is of divergence type, assumption (3.12) implies that $K_{\mu} \in L_{\text {loc }}^{p}(\mathbb{C})$ for every $1<p<\infty$. On the other hand, the inequality $D_{\mu, z_{0}}(z) \leq K(z)$ a.e. and the convergence of the integral (3.12) imply the local assumption (3.6). Hence, by Theorem [3.5, we have the required conclusion.

3.13. Remark. Condition (3.12) is optimal for the solvability of Beltrami equations in the following sense.

Assume that $H$ is a dominating factor of convergence type. We may assume that $H$ is smooth enough and $H(1)=1$. Then, by Theorem 3.1 of [12, there exists a $\mu$ satisfying (3.12) for which the following hold:

1. $\mu=0$ off the unit disk $B$,

2. $K_{\mu}$ is locally essentially bounded in $\mathbb{C} \backslash\{0\}$,

3. there are no $W_{\text {loc }}^{1,1}$-solutions to the Beltrami equation with $\mu$ in the unit disk which are continuous at the origin other than constant functions, and

4. there is a solution $f$ to the Beltrami equation in the weak- $W^{1,2}(B)$ Sobolev space, where weak- $W^{1,2}(B)=\bigcap_{1 \leq q<2} W^{1, q}(B)$, which maps the punctured disk $B \backslash\{0\}$ homeomorphically onto the annulus $A(0,1, R)$ for some $1<$ $R<+\infty$.

3.14. Corollary. Suppose that $\mu$ is a Beltrami coefficient in $\mathbb{C}$ such that for some $\eta>0$

$$
\int_{\mathbb{C}} e^{\eta K_{\mu}(z)} d \mathcal{A}^{\sharp}(z)<+\infty .
$$

Then there exists a $\mu$-conformal homeomorphism $f: \widehat{\mathbb{C}} \rightarrow \widehat{\mathbb{C}}$ such that $f \in W_{\text {loc }}^{1, q}(\mathbb{C})$ for every $q<2$ and $f^{-1} \in W_{\mathrm{loc}}^{1,2}(\mathbb{C})$. Moreover, for every compact set $E \subset \mathbb{C}$ there 
are positive constants $C, C^{\prime}$ and a such that

$$
C \exp \left(-\frac{a}{\left|z_{1}-z_{2}\right|^{2}}\right) \leq\left|f\left(z_{1}\right)-f\left(z_{2}\right)\right| \leq C^{\prime}\left|\log \frac{1}{\left|z_{1}-z_{2}\right|}\right|^{-\eta / 2}
$$

for $z_{1}, z_{2} \in E$ with $0<\left|z_{1}-z_{2}\right|<1 / e$. The exponent $-\eta / 2$ is sharp.

The right-hand side of (3.16) can be written in the more precise form

$$
\left|f\left(z_{1}\right)-f\left(z_{2}\right)\right| \leq C \operatorname{dist}\left(E_{0}, E_{1}\right)\left(\frac{1+\log \left(M / \pi R^{2}\right)}{\log 1 /\left|z_{1}-z_{2}\right|}\right)^{\eta / 2}
$$

for $z_{1}, z_{2} \in B(0, R / 2)$ with $\left|z_{1}-z_{2}\right|<1 / e$, where

$$
M=\int_{B(0, R)} e^{\eta K_{\mu}} d \mathcal{A}
$$

$E_{0}=f(\bar{B}(0, R / 2)), E_{1}=f(\widehat{\mathbb{C}} \backslash B(0, R))$ and $C$ is a constant depending only on $\eta$.

The sharpness can be seen by the following examples. Let $f: \mathbb{C} \rightarrow \mathbb{C}$ be the radial stretching defined by

$$
f(z)=\frac{z}{|z|}\left(1+\frac{2}{\beta} \log \frac{1}{|z|}\right)^{-\beta / 2}
$$

for $|z| \leq 1$ and $f(z)=z$ for $|z|>1$. (This example was given in [12, §11.1].) Then $K(z)=1+(2 / \beta) \log (1 /|z|)$ for $|z|<1$. Hence integrability condition (3.15) holds for $\beta>\eta$ but not for $\beta \leq \eta$. Since $|f(z)-f(0)|=\left(1+2 \beta^{-1} \log 1 /|z|\right)^{-\beta / 2} \sim$ $(\log 1 /|z|)^{-\beta / 2}$ as $z \rightarrow 0$, the exponent $\eta / 2$ in (3.16) cannot be replaced by any larger number.

3.18. Remark. It is noted in 3] that a necessary and sufficient condition for a measurable function $K(z) \geq 1$ to be majorized in $\Omega \subset \mathbb{C}$ by a function $M \in$ $B M O(\mathbb{C})$ is that

$$
\int_{\Omega} e^{\eta K(z)} d \mathcal{A}^{\sharp}(z)<+\infty
$$

for some positive number $\eta$. Moreover, $M$ can be chosen so that $\|M\|_{B M O} \leq C / \eta$, where $C$ is an absolute constant. For mappings of BMO-bounded distortion David [8] has proved the estimate

$$
\left|f\left(z_{1}\right)-f\left(z_{2}\right)\right| \leq A\left|\log \frac{1}{\left|z_{1}-z_{2}\right|}\right|^{-b /\|M\|_{B M O}}
$$

for some positive constant $b$, which agrees with our estimate (3.16).

3.21. Remark. It is shown in 12 that if $\mu$ has a compact support, then there exists a number $\eta_{0}>1$ such that the Beltrami equation with $\mu$ satisfying (3.15) with $\eta \geq \eta_{0}$ admits a unique principal solution $f$ with $f(z)-z \in W^{1,2}(\mathbb{C})$.

The following consequence is due to [12, Theorem 14.2] except for the modulus of continuity estimate. The almost same result has been earlier obtained in [6] (see Remark 3.26 below).

3.22. Corollary. Suppose that $\mu$ is a Beltrami coefficient in $\mathbb{C}$ such that

$$
\int_{\mathbb{C}} \exp \left\{\frac{\eta K_{\mu}(z)}{1+\log K_{\mu}(z)}\right\} d \mathcal{A}^{\sharp}(z)<\infty
$$


for some $\eta>0$. Then there exists a homeomorphic solution $f$ of (1.1) in $\widehat{\mathbb{C}}$ such that $f \in W_{\mathrm{loc}}^{1, q}(\mathbb{C})$ for every $q<2$ and $f^{-1} \in W_{\mathrm{loc}}^{1,2}(\mathbb{C})$. Moreover, for every compact set $E \subset \mathbb{C}$ there are constants $C, C^{\prime}$ and a such that

$$
C \exp \left\{-\frac{a}{\left|z_{1}-z_{2}\right|^{2}}\right\} \leq\left|f\left(z_{1}\right)-f\left(z_{2}\right)\right| \leq C^{\prime}\left(\log \log \frac{1}{\left|z_{1}-z_{2}\right|}\right)^{-\eta / 2}
$$

for $z_{1}, z_{2} \in E$ with $0<\left|z_{1}-z_{2}\right|<e^{-e}$. The exponent $-\eta / 2$ is sharp.

The modulus of continuity follows from the fact that $\eta^{-1} y \log y<H^{-1}(y)$ for sufficiently large $y$, where $H(x)=\eta x /\left(1+\log ^{+} x\right)$. More precisely, we have an estimate in the form

$$
\left|f\left(z_{1}\right)-f\left(z_{2}\right)\right| \leq C \operatorname{dist}\left(E_{0}, E_{1}\right)\left(\frac{\log \log \left(M / \pi R^{2}\right)}{\log \log \left(1 /\left|z_{1}-z_{2}\right|\right)}\right)^{\eta / 2}
$$

for $z_{1}, z_{2} \in B(0, R / 2)$ with $\left|z_{1}-z_{2}\right|<e^{-e}$, where

$$
M=\int_{B(0, R)} \exp \left\{\frac{\eta K_{\mu}(z)}{1+\log K_{\mu}(z)}\right\} d \mathcal{A}(z)
$$

$E_{0}=f(\bar{B}(0, R / 2)), E_{1}=f(\widehat{\mathbb{C}} \backslash B(0, R))$ and $C$ is a constant depending only on $\eta$.

Next we show the sharpness by examples. Let $f: \mathbb{C} \rightarrow \mathbb{C}$ be the radial stretching defined by

$$
f(z)=\frac{z}{|z|}\left(1+\frac{2}{\beta} \log \log \frac{e}{|z|}\right)^{-\beta / 2}
$$

for $|z| \leq 1$ and $f(z)=z$ for $|z|>1$. Then $K(z)=\log \frac{e}{|z|}\left(1+\frac{2}{\beta} \log \log \frac{e}{|z|}\right)$ for $|z|<1$. (This example appeared in [12, §12.1].) Hence

$$
\frac{K(z)}{1+\log K(z)}=\frac{2}{\beta} \log \frac{e}{|z|}\left(1-\frac{\log _{3} e /|z|}{\log _{2} e /|z|}+O\left(\frac{1}{\log _{2} e /|z|}\right)\right)
$$

as $z \rightarrow 0$ and, in particular, we see that $\exp \{\eta K(z) /(1+\log K(z))\}$ is integrable in $|z|<1$ if and only if $\beta \geq \eta$. Therefore the exponent $\eta / 2$ in (3.24) cannot be replaced by any larger number in general.

3.26. Remark. The first proof of the above statement was given in [6] under the assumption of special behavior of $K_{f}(z)$ around the point at infinity:

$$
\int_{B(0, R)} K(z) d \mathcal{A}(z)=O\left(R^{2}\right) .
$$

This assumption says that $K$ is bounded in the sense of the mean, and hence it is different from (3.23). Theorem 14.2 in [12] also contains the quite accurate regularity assertion

$$
\int_{\mathbb{C}} \frac{|\Psi(z)|^{2}}{\log (e+\Psi(z)) \log _{2}(3+\Psi(z))} d \mathcal{A}^{\sharp}(z)<+\infty,
$$

where $\Psi(z)$ stands for the spherical derivative $\frac{1+|z|^{2}}{1+|f(z)|^{2}}|D f(z)|$ of $f$.

Next, we prove Theorem 1.5 given in the Introduction, which shows that if $\operatorname{Sing}(\mu)$ of $\mu$ is a finite set, then $\mu$ can be modified without changing its absolute value and the modified $\mu$ admits a "good" solution. 
Proof. Let $\operatorname{Sing}(\mu)=\left\{a_{1}, \ldots, a_{n}\right\}$. Without loss of generality we will assume that all $a_{k}$ 's are finite. Let $\delta$ be a positive number such that the disks $\left|z-a_{k}\right| \leq \delta$, $k=1, \ldots, n$, are disjoint. Setting

$$
\mu^{\prime}(z)=|\mu(z)| \cdot \frac{z-a_{k}}{\bar{z}-\bar{a}_{k}}, \quad k=1, \ldots, n,
$$

for $\left|z-a_{k}\right| \leq \delta$ and $\mu^{\prime}(z)=\mu(z)$ otherwise, we see that $D_{\mu^{\prime}, z_{0}}(z)$ is essentially bounded in a sufficiently small disk $B\left(z_{0}, r_{0}\right)$ for each $z_{0} \in \widehat{\mathbb{C}}$. By Theorem 2.15 and Remark 2.18 there exists a homeomorphism $f: \widehat{\mathbb{C}} \rightarrow \widehat{\mathbb{C}}$ which is locally quasiconformal in $\Omega=\widehat{\mathbb{C}} \backslash\left\{a_{1}, \ldots, a_{n}\right\}$ and $\left.f\right|_{\Omega}$ satisfies the Beltrami equation with $\mu^{\prime}$. The condition $\left|\mu^{\prime}(z)\right|=|\mu(z)|$, which holds a.e., is immediate by construction.

3.27. Remark. For every $H \in \mathcal{D}$ there exists a homeomorphism $f: \widehat{\mathbb{C}} \rightarrow \widehat{\mathbb{C}}$ locally quasiconformal in $\widehat{\mathbb{C}} \backslash\left\{a_{1}, \ldots, a_{n}\right\}$ with complex dilatation $\mu$ such that

$$
\int_{\mathbb{C}} e^{H\left(K_{\mu}(z)\right)} d \mathcal{A}^{\sharp}(z)=+\infty
$$

but the weaker directional condition (3.6) holds.

In the above examples the singular set of $\mu$ consists of isolated points only. In the following examples the singular sets of $\mu$ are the whole extended real axis $\widehat{\mathbb{R}}=\mathbb{R} \cup\{\infty\}$.

3.28. Example. Define the Beltrami coefficient $\mu$ in $\widehat{\mathbb{C}}$ for $\alpha \in[1,2)$ as

$$
\mu(z)=1-\frac{2}{\alpha \log \frac{1}{|y|}}, \quad z=x+i y,
$$

for $|y| \leq y_{0}$ and as $\mu(z)=0$ for $|y|>y_{0}$, where $0<y_{0} \leq e^{-1 / \alpha}$ is a constant. Then integrability condition (3.15) with $\eta=1$ does not hold in any neighborhood of each point on the extended real axis, whereas condition (3.9) with $\eta=1$ still holds. Indeed, since $K_{\mu}(z)=1+\alpha \log (1 /|y|)$ for $|y|<y_{0}$, we see that $e^{K_{\mu}(z)}=e|y|^{-\alpha}$. Now the assumption $\alpha \geq 1$ implies that $e^{K_{\mu}}$ is not locally integrable at any point on the real axis. On the other hand, writing $z=r e^{i \theta}$, we compute for any $a \in \mathbb{R}$,

$$
D_{\mu, a}(z+a)=D_{\mu, 0}(z)=\frac{1-\mu}{1+\mu}+\frac{4 \mu}{1+\mu} \cdot \frac{\sin ^{2} \theta}{1-\mu} \leq 1+\alpha \sin ^{2} \theta\left(\log \frac{1}{|y|}\right),
$$

where the inequality $4 \mu /(1+\mu) \leq 2$ has been used. Since the function $x \log x$ is bounded in $(0,1)$, we obtain

$$
\exp \left(D_{\mu, a}(z+a)\right) \leq e|y|^{-\alpha \sin ^{2} \theta} \leq e r^{-\alpha} e^{-\frac{\alpha}{2} \sin ^{2} \theta \log \left(\sin ^{2} \theta\right)} \leq C r^{-\alpha}
$$

for some constant $C$. Hence, for sufficiently small $r_{0}>0$, we have

$$
\int_{B\left(a, r_{0}\right)} e^{D_{\mu, a}(z)} d \mathcal{A}^{\sharp}(z) \leq 2 \pi C \int_{0}^{r_{0}} r^{1-\alpha} d r<+\infty .
$$

Thus condition (3.9) holds for each $a \in$ Re. Similarly, we verify (3.9) for $a=\infty$.

3.29. Example. Another example of Beltrami coefficient $\mu$ in $\mathbb{C}$, for which $\operatorname{Sing}(\mu)=\widehat{\mathbb{R}}$, is provided by

$$
\mu(z)=1-\frac{2}{\alpha \log \frac{1}{|y|} \log \log \frac{1}{|y|}}, \quad z=x+i y,
$$


for $|y| \leq y_{0}$ and $\mu(z)=0$ for $|y|>y_{0}$, where $\alpha$ is a constant with $1<\alpha<$ $2 e /(e+1)=1.462 \ldots$ and $y_{0}$ is a positive constant with $y_{0} \leq e^{-e^{1 / \alpha}}$. An elementary computation shows that integrability assumption (3.23) does not hold at any neighborhood of each point lying on the real axis. On the other hand, assumption (3.10) still holds. Indeed, since $K_{\mu}(z)=1+\alpha \log (1 /|y|) \log _{2}(1 /|y|),|y|<y_{0}$, we obtain

$$
\frac{K_{\mu}(z)}{1+\log K_{\mu}(z)}=\frac{\alpha \log \frac{1}{|y|} \log _{2} \frac{1}{|y|}}{\log _{2} \frac{1}{|y|}+\log _{3} \frac{1}{|y|}+O(1)}=\alpha \log \frac{1}{|y|}\left[1+O\left(\frac{\log _{3} \frac{1}{|y|}}{\log _{2} \frac{1}{|y|}}\right)\right]
$$

as $y \rightarrow 0$. In particular, we see that $e^{K_{\mu}(z) /\left(1+\log K_{\mu}(z)\right)}$ is not locally integrable at every point on the real axis. On the other hand, as in the previous case, we obtain

$$
D_{\mu, 0}(z) \leq 1+\alpha \sin ^{2} \theta \log \frac{1}{|y|} \log _{2} \frac{1}{|y|}
$$

Hence, under the assumption that $\alpha \sin ^{2} \theta \log (1 /|y|) \log _{2}(1 /|y|)$ is large enough, we have

$$
\begin{aligned}
& \frac{D_{\mu, 0}(z)}{1+\log ^{+} D_{\mu, 0}(z)} \\
\leq & \frac{1+\alpha \sin ^{2} \theta \log \frac{1}{|y|}\left[\log \left(\alpha \sin ^{2} \theta \log \frac{1}{|y|} \log _{2} \frac{1}{|y|}\right)-\log \left(\alpha \sin ^{2} \theta \log _{2} \frac{1}{|y|}\right)\right]}{1+\log \left(\alpha \sin ^{2} \theta \log \frac{1}{|y|} \log _{2} \frac{1}{|y|}\right)} \\
= & 1+\alpha \sin ^{2} \theta \log \frac{1}{|y|}-\frac{\alpha \sin ^{2} \theta \log \frac{1}{|y|}\left[\log \left(\alpha \sin ^{2} \theta\right)+\log _{3} \frac{1}{|y|}\right]}{1+\log \left(\alpha \sin ^{2} \theta \log \frac{1}{|y|} \log _{2} \frac{1}{|y|}\right)} \\
\leq & \alpha \sin ^{2} \theta \log \frac{1}{|y|}+\frac{\alpha e^{-1} \log \frac{1}{|y|}}{1+\log \left(\alpha \sin ^{2} \theta \log \frac{1}{|y|} \log _{2} \frac{1}{|y|}\right)} \\
\leq & \alpha\left(\sin ^{2} \theta+e^{-1}\right) \log \frac{1}{|y|},
\end{aligned}
$$

where we used the inequality $-x \log x \leq e^{-1}$ for $0<x$. This yields

$$
\exp \left(\frac{D_{\mu, 0}(z)}{1+\log ^{+} D_{\mu, 0}(z)}\right) \leq|y|^{-\alpha\left(\sin ^{2} \theta+e^{-1}\right)} \leq C r^{-\alpha\left(1+e^{-1}\right)}|\sin \theta|^{-\alpha / e}
$$

for some positive constant $C$. The last function in the polar coordinates $(r, \theta)$ is integrable with respect to the measure $r d \theta d r$ over $0<r<1,0<|\theta|<\pi$ because $\alpha\left(1+e^{-1}\right)-1<1$ and $\alpha / e<1$. Thus, the function $\exp \left(D_{\mu, a} /\left(1+\log ^{+} D_{\mu, a}\right)\right)$ is integrable in a neighborhood of each $a \in$ Re. Similarly, we obtain the same assertion for $a=\infty$. Therefore, condition holds for each point $z_{0} \in \widehat{\mathbb{C}}$.

3.30. Remark. For the above examples, whose Beltrami coefficients $\mu(z)=$ $\mu(x+i y)$ depend on $y$ only, we can give normalized $\mu$-conformal homeomorphisms $f: \mathbb{C} \rightarrow \mathbb{C}$ in the explicit form

$$
f(z)=x+i \int_{0}^{y} \frac{1-\mu(i t)}{1+\mu(i t)} d t .
$$




\section{UNIQUENESS}

The following remark is a simple consequence of a well-known removability theorem for analytic functions.

4.1. Proposition. Let $\mu$ be a Beltrami coefficient in a domain $\Omega$ such that the singular set $E=\operatorname{Sing}(\mu)$ in $\Omega$ is countable. Suppose that a topological embedding $f: \Omega \rightarrow \widehat{\mathbb{C}}$ is locally quasiconformal in $\Omega \backslash E$ and satisfies (1.1). Then $f$ has the property that for any homeomorphic solution $\hat{f}$ of (1.1) in $\Omega \backslash E$ there exists a conformal map $h$ in $\Omega^{\prime}=f(\Omega)$ such that $\hat{f}=h \circ f$ in $\Omega \backslash E$. In particular, $\hat{f}$ extends to an embedding of $\Omega$.

Indeed, let us assume that $\hat{f}$ is another homeomorphic solution to the Beltrami equation (1.1) in $\Omega \backslash E$. Note that $\|\mu\|_{L^{\infty}\left(\Omega_{0}\right)}<1$ for every relatively compact open subset $\Omega_{0}$ of $\Omega \backslash E$. The local integrability of the Jacobian $J_{\hat{f}}=\left(1-|\mu|^{2}\right)\left|\hat{f}_{z}\right|^{2}$ of $\hat{f}$ (cf. the proof of Lemma 2.19) implies that $\hat{f} \in W_{\mathrm{loc}}^{1,2}(\Omega \backslash E)$. Since

$$
f^{-1} \in W_{\text {loc }}^{1,2}\left(\Omega^{\prime} \backslash f(E)\right),
$$

the function $h=\hat{f} \circ f^{-1}$ belongs to $W_{\text {loc }}^{1,1}\left(\Omega^{\prime} \backslash f(E)\right)$ and satisfies $h_{\bar{z}}=0$ there. Now Weyl's lemma yields that $h$ is an injective holomorphic map in $\Omega^{\prime} \backslash f(E)$. Since $f(E)$ is closed and countable in $\Omega^{\prime}$, it is removable for such a function and we conclude that $h$ can be extended to a conformal map on $\Omega^{\prime}$.

In order to apply the removability arguments for the uniqueness problem it seems necessary to have information on the singular set $E$ and its image $E^{\prime}=f(E)$, simultaneously; see, e.g., [13] and [3]. As Lehto [15] noted, it is reasonable to consider totally disconnected sets $E$. However, this condition does not imply uniqueness in general. We will introduce a geometric condition on totally disconnected compact sets $E$ and show that this condition can be combined with integrability conditions to guarantee the uniqueness.

A positive function $m(r)$ defined on the interval $(0, \delta)$ for small $\delta>0$ is said to be a modulus bound for a dominating factor $H$ of divergence type if it satisfies the condition

$$
\liminf _{r \rightarrow 0} \int_{0}^{m(r)} \frac{d t}{H^{-1}(2 t-2 \log r)}>0 .
$$

Note that $m(r) \rightarrow+\infty$ as $r \rightarrow 0$.

For example, if $H(x)=\eta x$, then $m(r)=\varepsilon \log (1 / r)$ is a modulus bound for $H$, where $\varepsilon$ is an arbitrary positive constant. If $H(x)=\eta x /\left(1+\log ^{+} x\right)$, then $m(r)=\varepsilon(\log (1 / r))^{C}$ is a modulus bound for $H$, where $\varepsilon>0$ and $C>1$ are arbitrary constants. More examples will be given in Section 5. Each modulus bound $m(r)$ generates the family of annuli $A_{m}\left(z_{0}, r\right)=A\left(z_{0}, r e^{-m(r)}, r\right)$ around every point $z_{0} \in \widehat{\mathbb{C}}$.

The following notions describe the thinness of the boundary. Let $H$ be a dominating factor. A compact subset $E$ of $\widehat{\mathbb{C}}$ is said to be $H$-coarse at $z_{0} \in E$ if there exists a modulus bound $m(r)$ for $H$ such that for any small number $\delta>0$ there is an $r$ with $0<r<\delta$ such that $A_{m}\left(z_{0}, r\right) \cap E=\emptyset$. The set $E$ is said to be $H$-coarse if $E$ is $H$-coarse at each point $z_{0} \in E$. We will also say that $E$ is radially coarse at $z_{0} \in E$ if a positive constant function can be chosen as $m(r)$ above, more precisely, there exists a positive number $m$ such that for any small number $\delta>0$ one can choose an $0<r<\delta$ with $A\left(z_{0}, r e^{-m}, r\right) \cap E=\emptyset$. 
It is clear that $z_{0} \in E$ forms a degenerate boundary component of $\Omega=\widehat{\mathbb{C}} \backslash E$ if $E$ is $H$-coarse at the point.

4.3. Theorem. Let $\mu$ be a Beltrami coefficient in $\widehat{\mathbb{C}}$ such that $E=\operatorname{Sing}(\mu)$ is a totally disconnected compact subset of $\widehat{\mathbb{C}}$. Assume that one of the following conditions holds for each $z_{0} \in E$ :

1) $D_{\mu, z_{0}}(z)$ is essentially bounded in a neighborhood of $z_{0}$ and $E$ is radially coarse at $z_{0}$;

2) There is a dominating factor $H=H_{z_{0}}$ of divergence type for which $E$ is $H$-coarse at $z_{0}$ and

$$
\int_{V} e^{H\left(D_{\mu, z_{0}}(z)\right)} d \mathcal{A}^{\sharp}(z)<+\infty
$$

for some open neighborhood $V$ of $z_{0}$.

Then there exists a homeomorphism $f: \widehat{\mathbb{C}} \rightarrow \widehat{\mathbb{C}}$ which is locally quasiconformal in $\Omega=\widehat{\mathbb{C}} \backslash E$ and satisfies the Beltrami equation with $\mu$ in $\Omega$. If $\hat{f}$ is another homeomorphic solution to the Beltrami equation in $\Omega$ with the same $\mu$, then $\hat{f}=h \circ f$ for a Möbius transformation $h$. In particular, $\hat{f}$ can be extended to a homeomorphism of $\widehat{\mathbb{C}}$.

For the proof of this theorem we need the following auxiliary lemma which is due to Gotoh and Taniguchi. A compact set $E$ is said to be annularly coarse at $z_{0} \in E$ if we can find a nesting sequence of disjoint ring domains $A_{n}$ around $z_{0}$ in $\widehat{\mathbb{C}} \backslash E$ with $\inf _{n} \bmod A_{n}>0$. Here a sequence of disjoint ring domains $A_{n}$ is said to be nesting if each $A_{n}$ separates $A_{n-1}$ from $A_{n+1}$. A compact set $E$ is said to be annularly coarse if $E$ is annularly coarse at every point in $E$.

4.5. Lemma (Gotoh-Taniguchi [10]). If a compact subset E of the Riemann sphere is annularly coarse, then $E$ is removable for conformal mappings off $E$.

Proof of Theorem 4.3. Let $f$ be a uniform limit of the canonical approximating sequence for $\mu$. Note that the existence of such an $f$ is guaranteed by Theorem 2.15. Set $E^{\prime}=f(E)$. We show that $E^{\prime}$ is annularly coarse. We may assume that $E \subset \mathbb{C}$. Let $z_{0} \in E$. Suppose first that case 2) occurs for $z_{0}$. Then, by assumption, there is an $H \in \mathcal{D}$ and a constant $M$ with

$$
\int_{B\left(z_{0}, r_{0}\right)} e^{H\left(D_{\mu, z_{0}}(z)\right)} d \mathcal{A}(z) \leq M
$$

for which $E$ is $H$-coarse at $z_{0}$ with modulus bound $m:(0, \delta) \rightarrow(0,+\infty)$. We then take arbitrarily small $r>0$ so that $A_{m}\left(z_{0}, r\right) \subset \Omega$. By Lemma 2.22 , we estimate

$$
\bmod f\left(A_{m}\left(z_{0}, r\right)\right) \geq \int_{1 / 2}^{m(r)} \frac{d t}{H^{-1}\left(2 t+\log \left(M / \pi r^{2}\right)\right)} .
$$

It is easy to see that

$$
\int_{a}^{b} \frac{d t}{H^{-1}(2 t+c-2 \log r)} \rightarrow 0
$$

as $r \rightarrow 0$ for any fixed $a, b$ and $c$. Hence, by the definition of the modulus bound, we obtain

$$
\liminf _{r \rightarrow 0} \int_{1 / 2}^{m(r)} \frac{d t}{H^{-1}\left(2 t+\log \left(M / \pi r^{2}\right)\right)}=\liminf _{r \rightarrow 0} \int_{0}^{m(r)} \frac{d t}{H^{-1}(2 t-2 \log r)}>0 .
$$


We can now find a sequence $r_{n}$ with $0<r_{n+1}<r_{n} e^{-m\left(r_{n}\right)}$ such that $A_{m}\left(z_{0}, r_{n}\right) \subset \Omega$ for $n=1,2, \ldots$ and that $\inf _{n} \bmod f\left(A_{m}\left(z_{0}, r_{n}\right)\right)>0$. Hence, we conclude that $E^{\prime}$ is annularly coarse at $f\left(z_{0}\right)$.

If case 1 ) occurs for $z_{0}$, then $E$ is radially coarse at $z_{0}$. Now it easy to see that $E^{\prime}$ is annularly coarse at $f\left(z_{0}\right)$ (cf. Lemma 2.19).

Assume that $\hat{f}$ is another homeomorphic solution to the Beltrami equation (1.I) with $\mu$ in $\Omega$. Then the function $h=\hat{f} \circ f^{-1}$ is an injective holomorphic function in $\widehat{\mathbb{C}} \backslash E^{\prime}$. Since $E^{\prime}=f(E)$ is removable for such a function by Lemma 4.5, we conclude that $h$ extends to a Möbius transformation. Thus, the proof is complete.

Specifying $H \in \mathcal{D}$, we obtain some consequences of Theorem 4.3. We only consider the dominating factors $H(x)=\eta x$ and $H(x)=\eta x /\left(1+\log ^{+} x\right)$ corresponding to exponential and subexponential integrability assumptions on $K_{\mu}(z)$, respectively.

4.6. Corollary. Let $E$ be a totally disconnected, $x$-coarse compact subset of $\widehat{\mathbb{C}}$. Suppose that $\mu$ is a Beltrami coefficient in $\mathbb{C}$ with $\operatorname{Sing}(\mu) \subset E$ such that

$$
\int_{\mathbb{C}} e^{\eta K_{\mu}(z)} d \mathcal{A}^{\sharp}(z)<\infty
$$

holds for a positive constant $\eta$. Then there exists a homeomorphism $f: \widehat{\mathbb{C}} \rightarrow \widehat{\mathbb{C}}$ which is locally quasiconformal in $\Omega=\widehat{\mathbb{C}} \backslash E$ and satisfies the Beltrami equation with $\mu$ in $\Omega$. If $\hat{f}: \Omega \rightarrow \widehat{\mathbb{C}}$ is a locally quasiconformal embedding whose Beltrami coefficient agrees with $\mu$ a.e., then $\hat{f}=h \circ f$ for a Möbius transformation $h$. In particular, $\hat{f}$ extends to a homeomorphism of the Riemann sphere.

4.8. Corollary. Let $E$ be a totally disconnected compact subset of $\widehat{\mathbb{C}}$ which is $x /\left(1+\log ^{+} x\right)$-coarse in E. Suppose that $\mu$ is a Beltrami coefficient in $\mathbb{C}$ with $\operatorname{Sing}(\mu) \subset E$ such that

$$
\int_{\mathbb{C}} \exp \left\{\frac{\eta K_{\mu}(z)}{1+\log K_{\mu}(z)}\right\} d \mathcal{A}^{\sharp}(z)<\infty .
$$

Then there exists a homeomorphism $f: \widehat{\mathbb{C}} \rightarrow \widehat{\mathbb{C}}$ which is locally quasiconformal in $\Omega=\widehat{\mathbb{C}} \backslash E$ and satisfies the Beltrami equation with $\mu$ in $\Omega$. If $\hat{f}: \Omega \rightarrow \widehat{\mathbb{C}}$ is a locally quasiconformal embedding whose Beltrami coefficient agrees with $\mu$ a.e., then $\hat{f}=$ $h \circ f$ for a Möbius transformation $h$. In particular, $\hat{f}$ extends to a homeomorphism of the Riemann sphere.

\section{Dominating FACTORS AND MODUlus BOUNDS}

In this section, we provide critical dominating factors $H$ of divergence type and their modulus bounds. The functions presented below are more or less standard. For example, similar examples can be found in [12].

We define the functions $\log _{n}, \exp _{n}, \Pi_{n, \alpha}$ and $\Pi_{n}$ for $n=0,1,2, \ldots$ and for $\alpha>0$ by

$$
\begin{gathered}
\log _{0} x=x, \quad \log _{n} x=\log \left(\log _{n-1} x\right) \quad(n=1,2, \ldots), \\
\exp _{0} x=x, \quad \exp _{n}=\exp \left(\exp _{n-1} x\right) \quad(n=1,2, \ldots), \\
\Pi_{n, \alpha}(x)=x\left(\log _{1} x\right) \cdots\left(\log _{n-1} x\right)\left(\log _{n} x\right)^{\alpha}, \text { and } \\
\Pi_{n}(x)=\Pi_{n, 1}(x) \quad(n=0,1,2, \ldots) .
\end{gathered}
$$


In particular, $\log _{1}$ and $\exp _{1}$ coincide with the standard log and exp, respectively. Note that $\log _{n}$ is the inverse function of $\exp _{n}$ for each $n$. We also define the numbers $e_{n}$ by $e_{n}=\exp _{n} 0$. Then for each $n>0$ the functions $\log _{n}, \Pi_{n, \alpha}$ and $\Pi_{n}$ are defined on $\left(e_{n-1},+\infty\right)$, positive on $\left(e_{n},+\infty\right)$, and greater than 1 on $\left(e_{n+1},+\infty\right)$.

We consider the function $H(x)=x^{2} / \Pi_{n, \alpha}(x)$ for fixed $n \geq 0$ and $\alpha>0$. Then, $H^{\prime}(x)=(1+o(1)) H(x) / x$ and $H^{\prime \prime}(x)=(1+o(1)) H(x) / \log x$ when $x \rightarrow+\infty$. In particular, $\left(e^{H}\right)^{\prime \prime} / e^{H}=H^{\prime \prime}+\left(H^{\prime}\right)^{2}=(1+o(1))\left(H^{\prime}\right)^{2}$. Hence, we can choose a sufficiently large number $x_{n, \alpha}>e_{n}$ so that the function $H_{n, \alpha}$ defined by

$$
H_{n, \alpha}(x)=\left\{\begin{array}{cl}
H(x)=\frac{x^{2}}{\Pi_{n, \alpha}(x)} & \text { if } x>x_{n, \alpha}, \\
H\left(x_{n, \alpha}\right) & \text { if } 0 \leq x \leq x_{n, \alpha},
\end{array}\right.
$$

is a dominating factor. Furthermore, $H_{n, \alpha}$ is of divergence type if and only if $\alpha \leq 1$ since the integral

$$
\int_{x_{n, \alpha}}^{\infty} \frac{H_{n, \alpha}(x) d x}{x^{2}}=\int_{x_{n, \alpha}}^{\infty} \frac{d x}{\Pi_{n, \alpha}(x)}=\int_{x_{n, \alpha}}^{\infty} \frac{d x}{x(\log x) \ldots\left(\log _{n-1} x\right)\left(\log _{n} x\right)^{\alpha}}
$$

is divergent if and only if $\alpha \leq 1$. Note that the choice of $x_{n, \alpha}$ does not effect the integrability condition such as $\int_{V} e^{H\left(D_{\mu, z_{0}}\right)} d \mathcal{A}^{\sharp}<+\infty$. We also write $H_{n}=H_{n, 1}$.

5.1. Remark. The dominating factor $H_{0}=H_{0,1}$ is essentially the function $x$. The dominating factor $H_{1}=H_{1,1}$ is equivalent to $H(x)=x /\left(1+\log ^{+} x\right)$ in the sense that for any $\eta>1$ we have $H(x)<H_{1}(x)<\eta H(x)$ for sufficiently large $x$.

First we give information on the behavior of the inverse function of $H_{n, \alpha}$.

5.2. Lemma. For each $n \geq 1, \alpha \in \mathbb{R}$ and $c>0$, the estimate

$$
\left(c H_{n, \alpha}\right)^{-1}(y)=\frac{\Pi_{n, \alpha}(y)}{c}\left(1+(b+o(1)) \frac{\log _{2} y}{\log y}\right) \quad(y \rightarrow+\infty)
$$

holds, where $b=1$ when $n>1$ and $b=\alpha^{2}$ when $n=1$. Suppose further that $\alpha \leq 1$ and, in addition if $n=1$, suppose that $0 \leq \alpha$. Then

$$
\int_{t_{1}}^{t} \frac{d y}{\left(c H_{n, \alpha}\right)^{-1}(y)}=\left\{\begin{array}{cl}
\frac{c}{1-\alpha}\left(\log _{n} t\right)^{1-\alpha}+O(1) & \text { when } \alpha<1, \\
c \log _{n+1} t+O(1) & \text { when } \alpha=1
\end{array}\right.
$$

as $t \rightarrow+\infty$ for a sufficiently large number $t_{1}$. When $n=1$ and $\alpha<0$,

$$
\int_{t_{1}}^{t} \frac{d y}{\left(c H_{1, \alpha}\right)^{-1}(y)}=\left(\frac{c}{1-\alpha}+o(1)\right)(\log t)^{1-\alpha}
$$

as $t \rightarrow+\infty$ for a sufficiently large number $t_{1}$.

Proof. We assume that $n \geq 2$. (The same and somewhat easier method can be used for $n=1$.) Letting $\eta=\eta(y)$ be a positive quantity with $\log \eta(y)=O(1)$ as $y \rightarrow \infty$, we observe

$$
\begin{aligned}
\log \left(\eta \Pi_{n, \alpha}(y)\right) & =\log \eta+\log y+\log _{2} y+\cdots+\log _{n} y+\alpha \log _{n+1} y \\
& =\left(1+(1+o(1)) \frac{\log _{2} y}{\log y}\right) \log y \quad(y \rightarrow+\infty)
\end{aligned}
$$


Inductively we then obtain that

$$
\log _{k}\left(\eta \Pi_{n, \alpha}(y)\right)=\log _{k} y \cdot\left(1+(1+o(1)) \log _{2} y / \log y \log _{2} y \cdots \log _{k} y\right)
$$

for every integer $k \geq 1$. Therefore,

$$
\begin{aligned}
c H_{n, \alpha}\left(\eta \Pi_{n, \alpha}(y)\right) & =\frac{c \eta \Pi_{n, \alpha}(y)}{\log \left(\eta \Pi_{n, \alpha}(y)\right) \cdots\left(\log _{n}\left(\eta \Pi_{n, \alpha}(y)\right)\right)^{\alpha}} \\
& =c \eta y\left(1-(1+o(1)) \frac{\log _{2} y}{\log y}\right)
\end{aligned}
$$

as $y \rightarrow+\infty$. We now restrict ourselves to the case when $c \eta=1+p \log _{2} y / \log y$ for some constant $p$. Then $\left(1-\log _{2} y / \log y\right) c \eta=1+(p-1)(1+o(1)) \log _{2} / \log y$ and since $\eta \Pi_{n, \alpha}(y)>H_{n, \alpha}{ }^{-1}(y)$ for sufficiently large $y$ if $p>1$ and $\eta \Pi_{n, \alpha}(y)<H_{n, \alpha}{ }^{-1}(y)$ for sufficiently large $y$ if $p<1$, the asymptotic formula for the inverse function follows.

We next show the second assertion. For simplicity, we assume that $\alpha<1$; the case $\alpha=1$ can be absorbed to this case because $H_{n, 1}(y)=H_{n+1,0}(y)$ for large $y$. By the first assertion, we obtain

$$
\int_{t_{1}}^{t} \frac{d y}{\left(c H_{n, \alpha}\right)^{-1}(y)}=\int_{t_{1}}^{t} \frac{c d y}{\Pi_{n, \alpha}(y)}+(-b c+o(1)) \int_{t_{1}}^{t} \frac{\log _{2} y d y}{y(\log y)^{2} \log _{2} y \cdots\left(\log _{n} y\right)^{\alpha}}
$$

if $n>1$. For $n=1$, a slight modification is needed. Since the second integral in the right-hand side is convergent as $t \rightarrow+\infty$ under the assumptions on $\alpha$, the required asymptotic formula is obtained.

Proof of Theorem 1.6. Without loss of generality, we may assume that $\alpha<1$ (see the proof of Lemma (5.2). Under the hypothesis, the integrability condition

$$
\int_{\mathbb{C}} e^{c H_{n, \alpha}\left(K_{\mu}(z)\right)} d \mathcal{A}^{\sharp}(z)<\infty
$$

holds. Since $\mathrm{cH}_{n, \alpha}$ is a dominated factor of divergence type, we conclude from Theorem 3.11 the existence and the regularity of the normalized homeomorphic solution $f$ to the Beltrami equation. We now investigate the modulus of continuity of $f$. From the assumption, for an arbitrary number $R>1$, it follows that

$$
\int_{B\left(z_{0}, 1\right)} e^{c H_{n, \alpha}\left(K_{\mu}(z)\right)} d \mathcal{A}(z) \leq M=\int_{B(0, R+1)} e^{c H_{n, \alpha}\left(K_{\mu}(z)\right)} d \mathcal{A}(z)<\infty
$$

for each point $z_{0}$ with $\left|z_{0}\right| \leq R$. Setting $b=\log (M / \pi)$, we obtain from Theorem 3.5 that

$$
\left|f(z)-f\left(z_{0}\right)\right| \leq C \exp \left\{-\frac{1}{2} \int_{1+b}^{2 m+b} \frac{d t}{\left(c H_{n, \alpha}\right)^{-1}(t)}\right\}, \quad\left|z-z_{0}\right|<1,
$$

where $m=\log \left(1 /\left|z-z_{0}\right|\right)$ and $C$ is a constant depending only on $\mu, c, n, \alpha$ and $R$. Applying Lemma 5.2, in the case when $n>1$, we have

$$
\begin{aligned}
\frac{1}{2} \int_{1+b}^{2 m+b} \frac{d t}{\left(c H_{n, \alpha}\right)^{-1}(t)} & =\frac{c}{2(1-\alpha)}\left(\log _{n}(2 m+b)\right)^{1-\alpha}+O(1) \\
& =\frac{c}{2(1-\alpha)}\left(\log _{n}(m)\right)^{1-\alpha}+O(1)
\end{aligned}
$$

as $m \rightarrow+\infty$. The case when $n=1$ follows similarly from the latter part of Lemma 5.2. Thus we have shown the estimate for the modulus of continuity of $f$. 
Next we construct an example; see the discussion after Theorem 1.6. As we noted, a more abstract approach for a dominated factor of convergence type can be found in [12, Theorem 3.1].

Let $M(t)=\eta \log (1 / t) \cdots \log _{n}(1 / t)\left(\log _{n+1}(1 / t)\right)^{\alpha}=\eta \Pi_{n, \alpha}(\log (1 / t))$, where $\eta>$ $0, n \geq 0$ and $\alpha \in \mathbb{R}$. Let $f$ be the radial stretching

$$
f(z)=\frac{z}{|z|} \exp \int_{\delta_{0}}^{|z|} \frac{d t}{t M(t)}
$$

in $0<|z|<\delta_{0}$, where $\delta_{0}$ is a sufficiently small number. Then, as is easily seen, the pointwise maximal dilatation of $f$ is given by $K(z)=M(|z|)$. Note that the function $f$ can be continuously extended to $z=0$ by setting $f(0)=0$ if and only if the integral $\int_{0}^{\delta_{0}} d t / t M(t)$ diverges, namely, $\alpha \leq 1$. In particular, when $\alpha>1$, by Theorem 2.15 (and Proposition 4.1 as well), $\exp (H \circ K(z))$ is not locally integrable around the origin for every dominating factor $H$ of divergence type. On the other hand, we see that $H \circ K$ is exponentially integrable around the origin for $H=c H_{n, \alpha}$, where $c$ is a constant satisfying $c<2 / \eta$. We fix a number $\varepsilon$ with $c \eta<\varepsilon<2$. From (5.3), it follows that $H(M(t))=c H_{n, \alpha}\left(\eta \Pi_{n, \alpha}(\log (1 / t))\right)=c \eta(1+o(t)) \log (1 / t)$ as $t \rightarrow+0$. Therefore, there is a number $r_{0}>0$ such that $H(M(t)) \leq \varepsilon \log (1 / t)$ for all $0<t<r_{0}$. We now compute

$$
\int_{B\left(0, r_{0}\right)} e^{H(K(z))} d \mathcal{A}(z)=2 \pi \int_{0}^{r_{0}} t e^{H(M(t))} d t \leq 2 \pi \int_{0}^{r_{0}} t^{1-\varepsilon} d t<+\infty .
$$

In particular, the exponential integrability condition for $H_{n, \alpha} \circ K_{\mu}$ does not imply the existence of a homeomorphic solution in the case $\alpha>1$.

Next, we assume that $\alpha<1$. (As we noted, the case $\alpha=1$ can be included in the case $\alpha=0$.) Then, the function $f$ can be expressed in the form

$$
f(z)=\frac{C z}{|z|} \exp \left(\frac{-1}{(1-\alpha) \eta}\left(\log _{n+1}(1 /|z|)\right)^{1-\alpha}\right), \quad 0<|z|<\delta_{0} .
$$

Since $\eta$ can be chosen arbitrarily whenever $\eta<2 / c$, the sharpness is obtained.

To state results on modulus bounds of the above dominating factors, we present some auxiliary functions. For constants $C>1$ and $\beta, \delta>0$ and a non-negative integer $n$, we set

$$
\begin{aligned}
\varphi_{n, C}(x) & =\exp _{n}\left(\log _{n} x+\log C\right) \text { and } \\
\psi_{n, \beta, \delta}(x) & =\exp _{n}\left(\left[\left(\log _{n} x\right)^{\beta}+\delta\right]^{1 / \beta}\right)
\end{aligned}
$$

for $x \geq e_{n}$. By definition, $\varphi_{n, C}=\psi_{n, 1, \log C}$, and for example

$$
\begin{aligned}
\varphi_{0, C}(x) & =x+\log C, \\
\varphi_{1, C}(x) & =C x, \text { and } \\
\varphi_{2, C}(x) & =x^{C} .
\end{aligned}
$$

5.4. Proposition. For a positive integer $n$ a modulus bound for $H_{n}$ is given by

$$
m(r)=\varepsilon \varphi_{n+1, C}(\log 1 / r)
$$

for $r$ small enough, where $\varepsilon>0$ and $C>0$ are constants. 
Proof. From Lemma 5.2, we see that

$$
\begin{aligned}
& \liminf _{r \rightarrow 0} \int_{0}^{m(r)} \frac{d t}{H_{n}{ }^{-1}(2 t-2 \log r)} \\
= & \frac{1}{2} \liminf _{r \rightarrow 0}\left[\log _{n+1}(2 m(r)-2 \log r)-\log _{n+1}(-2 \log r)\right] .
\end{aligned}
$$

Noting that $\log r=o(m(r))$ as $r \rightarrow 0$, we obtain

$$
\begin{aligned}
\log _{n+1}(2 m(r)-2 \log r) & =\log _{n+1}\left(\varphi_{n+1, C}(\log 1 / r)\right)+o(1) \\
& =\log _{n+1}(\log 1 / r)+\log C+o(1)
\end{aligned}
$$

and $\log _{n+1}(m(r))=\log _{n+1}(\log 1 / r)+o(1)$ as $r \rightarrow 0$. Hence, $m(r)$ is a modulus bound for $H_{n}$.

The same method yields the following result.

5.5. Proposition. For a positive integer $n$ and $\alpha \in(0,1)$, a modulus bound for $H_{n, \alpha}$ is given by

$$
m(r)=\varepsilon \psi_{n, 1-\alpha, \delta}(\log 1 / r)
$$

for $r$ small enough, where $\varepsilon>0$ and $\delta>0$ are constants.

\section{ACKNOWLEDGEMENT}

This research was carried out during the first and the third authors' visit to the University of Helsinki. These authors are thankful to the Department of Mathematics for its financial support and hospitality.

\section{REFERENCES}

[1] L. V. Ahlfors, Lectures on Quasiconformal Mappings, van Nostrand, 1966. MR0200442 $(34: 336)$

[2] C. Andreian Cazacu, Influence of the orientation of the characteristic ellipses on the properties of the quasiconformal mappings, Proceedings of the Romanian-Finnish Seminar on Teichmüller Spaces and Quasiconformal Mappings (Braşov, 1969), Publ. House of the Acad. of the Socialist Republic of Romania, Bucharest, 1971, pp. 65-85. MR 0318478 (47:7025)

[3] K. Astala, T. Iwaniec, P. Koskela, and G. J. Martin, Mappings of BMO-bounded distortion, Math. Ann. 317 (2000), 703-726. MR.1777116|(2001i:30016)

[4] P. P. Belinskiǔ, General properties of quasiconformal mappings (Russian), Izdat. "Nauka" Sibirsk. Otdel., Novosibirsk, 1974. MR0407275 (53:11054)

[5] L. Bers, Uniformization by Beltrami equation, Commun. in Pure and Appl. Math. 14 (1961), 215-228. MR0132175 (24:A2022)

[6] M. A. Brakalova and J. A. Jenkins, On solutions of the Beltrami equation, J. Anal. Math. 76 (1998), 67-92. MR1676936 (2000h:30029)

[7] Z.-G. Chen, Estimates on $\mu(z)$-homeomorphisms of the unit disk, Israel J. Math. 122 (2001), 347-358. MR 1826507 (2002b:30020)

[8] G. David, Solutions de l'équation de Beltrami avec $\|\mu\|_{\infty}=1$, Ann. Acad. Sci. Fenn. Ser. A I Math. 13 (1988), 25-70. MR0975566 (90d:30058)

[9] F. W. Gehring, A remark on the moduli of rings, Comment. Math. Helv. 36 (1962), 42-46. MR.0140682(25:4097)

[10] Y. Gotoh and M. Taniguchi, A condition of quasiconformal extendability, Proc. Japan Acad. Ser. A Math. Sci. 75 (1999), 58-60. MR1701526|(2000e:30037)

[11] D. A. Herron, X. Liu, and D. Minda, Ring domains with separating circles or separating annuli, J. Anal. Math. 53 (1989), 233-252. MR.1014988 (91i:30018)

[12] T. Iwaniec and G. Martin, The Beltrami equation - In memory of Eugenio Beltrami (18351900), 100 years on, Memoires of the AMS, American Mathematical Society, to appear. 
[13] V. I. Kruglikov, The existence and uniqueness of mappings that are quasiconformal in the mean, Metric questions of the theory of functions and mappings, No. IV (Russian), Izdat. "Naukova Dumka", Kiev, 1973, pp. 123-147. MR0344462 (49:9201)

[14] O. Lehto, Homeomorphisms with a given dilatation, Proceedings of the Fifteenth Scandinavian Congress (Oslo, 1968), Springer, 1970, pp. 58-73. MR0260997 (41:5617)

[15] - Remarks on generalized Beltrami equations and conformal mappings, Proceedings of the Romanian-Finnish Seminar on Teichmüller Spaces and Quasiconformal Mappings (Braşov, 1969), Publ. House of the Acad. of the Socialist Republic of Romania, Bucharest, 1971, pp. 203-214. MR0306489 (46:5615)

[16] O. Lehto and K. I. Virtanen, Quasiconformal Mappings in the Plane, 2nd Ed., SpringerVerlag, 1973. MR0344463 (49:9202)

[17] V. G. Maz'ja, Sobolev spaces, Springer-Verlag, Berlin, 1985, Translated from the Russian by T. O. Shaposhnikova. MR0817985 (87g:46056)

[18] V. M. Miklyukov and G. D. Suvorov, The existence and uniqueness of quasiconformal mappings with unbounded characteristics (Russian), Studies in the theory of functions of a complex variable and its applications, Vidannja Inst. Mat. Akad. Nauk Ukraïn. RSR, Kiev, 1972, pp. 45-53. MR0338361|(49:3126)

[19] I. N. Pesin, Mappings which are quasiconformal in the mean (Russian), Dokl. Akad. Nauk SSSR 187 (1969), 740-742, English translation in Soviet Math. Dokl. 10 (1969), 939-941. MR $0249613(40: 2856)$

[20] E. Reich and H. Walczak, On the behavior of quasiconformal mappings at a point, Trans. Amer. Math. Soc. 117 (1965), 338-351. MR0176070 (31:345)

[21] V. Ryazanov, U. Srebro, and E. Yakubov, BMO-quasiregular mappings, J. Anal. Math. 83 (2001), 1-20. MR1828484 (2002a:30034)

[22] U. Srebro and E. Yakubov, $\mu$-homeomorphisms, Lipa's legacy (New York, 1995) (Providence, RI), Amer. Math. Soc., Providence, RI, 1997, pp. 473-479. MR1477004 (99c:30043)

[23] G. D. Suvorov, Families of plane topological mappings (Russian), Izdat. Sibirsk. Otdel. Akad. Nauk SSSR, Novosibirsk, 1965. MR0199425|(33:7570)

[24] P. Tukia, Compactness properties of $\mu$-homeomorphisms, Ann. Acad. Sci. Fenn. Ser. A I Math. 16 (1991), 47-69. MR1127696 (93c:30029)

Institute of Applied Mathematics and Mechanics, NAS of Ukraine, ul. Roze LuxemBURG 74, 83114, Donetsk, Ukraine

E-mail address: gut@iamm.ac.donetsk.ua

Department of Mathematics, P.O. Box 68 (Gustaf Hällströmin katu 2B), Fin-00014 University of Helsinki, Finland

E-mail address: martio@cc.helsinki.fi

Department of Mathematics, Graduate School of Science, Hiroshima University, 739 - 8526 Higashi-Hiroshima, JAPAN

E-mail address: sugawa@math.sci.hiroshima-u.ac.jp

Department of Mathematics, FIN-20014 University of Turku, Finland

E-mail address: vuorinen@csc.fi 